\title{
Development and thorough characterization of the processing steps of an ink for 3D printing for bone tissue engineering
}

Michael Müller, ${ }^{a}$ Philipp Fisch ${ }^{a}$, Marc Molnar ${ }^{a}$, Sebastian Eggert ${ }^{a}$, Marco Binelli ${ }^{a}$, Katharina ManiuraWeber $^{b}$, Marcy Zenobi-Wong ${ }^{\mathrm{a} *}$

a) Tissue Engineering + Biofabrication Laboratory, ETH Zürich, Otto-Stern-Weg 7, 8093 Zürich, Switzerland

b) Laboratory for Biointerfaces, Swiss Federal Laboratories for Materials Science and Technology (Empa), Lerchenfeldstrasse 5, 9014 St. Gallen, Switzerland

*Corresponding author. Marcy Zenobi-Wong, Department of Health Science \& Technology, Tissue Engineering + Biofabrication, Otto-Stern-Weg 7, Office HPL J22, 8093 Zürich, Switzerland. Phone: +41 4463250 89, Fax: +41 4463311 94, email: marcy.zenobi@hest.ethz.ch;

Keywords: Biomaterial Ink Processing, Biomaterial Ink Characterization, Biphasic Constructs, Tissue Engineering, 3D Printing

This document is the accepted manuscript version of the following article: Mü11er, M., Fisch, P., Molnar, M., Eggert, S., Bine11i, M., Maniura-Weber, K., \& Zenobi-Wong, M. (2019). Development and thorough characterization of the processing steps of an ink for 3D printing for bone tissue engineering. Materials Science and Engineering C: Biomimetic Materials, Sensors and Systems, 110510 (38 pp.). https://doi.org/10.1016/j.msec.2019.110510 


\section{Highlights}

- Synthetic approach for gellan gum methacrylate influences rheological properties

- Extrusion force measurements successfully used to predict printability of inks

- Thermogravimetric analysis revealed even distribution of HAp particles throughout cartridge

- Mesenchymal stromal cells adhere upon introduction of hydroxyapatite to ink 


\section{Abstract}

Achieving reproducibility in the 3D printing of biomaterials requires a robust polymer synthesis method to reduce batch-to-batch variation as well as methods to assure a thorough characterization throughout the manufacturing process. Particularly biomaterial inks containing large solid fractions such as ceramic particles, often required for bone tissue engineering applications, are prone to inhomogeneity originating from inadequate mixing or particle aggregation which can lead to inconsistent printing results. The production of such an ink for bone tissue engineering consisting of gellan gum methacrylate (GG-MA), hyaluronic acid methacrylate and hydroxyapatite (HAp) particles was therefore optimized in terms of GG-MA synthesis and ink preparation process, and the ink's printability was thoroughly characterized to assure homogeneous and reproducible printing results. A new buffer mediated synthesis method for GG-MA resulted in consistent degrees of substitution which allowed the creation of large $5 \mathrm{~g}$ batches. We found that both the new synthesis as well as cryomilling of the polymer components of the ink resulted in a decrease in viscosity from $113 \mathrm{kPa} \cdot \mathrm{s}$ to $11.3 \mathrm{kPa} \cdot \mathrm{s}$ at a shear rate of $0.1 \mathrm{~s}^{-1}$ but increased ink homogeneity. The ink homogeneity was assessed through thermogravimetric analysis and a newly developed extrusion force measurement setup. The ink displayed strong inter-layer adhesion between two printed ink layers as well as between a layer of ink with and a layer without HAp. The large polymer batch production along with the characterization of the ink during the manufacturing process allows ink production in the gram scale and could be used in applications such as the printing of osteochondral grafts. 


\section{Introduction}

Tissue engineering has emerged over the last decades due to a high need to repair or replace diseased and injured tissues and organs. In tissue engineering, cells are combined with growth factors and scaffolds which provide physical support for the cells and aid with the formation of a three-dimensional (3D) tissue [1]. Conventionally, fabrication methods such as particle leaching [2], gas foaming [3] or electrospinning [4] are used to create scaffolds for tissue engineering. However, these aforementioned scaffold fabrication techniques do not offer precise control over the porosity, pore interconnectivity or spatial distribution of the pores. 3D printing has recently attracted much attention due to the fact that it has the potential to overcome the shortages of traditionally employed scaffold fabrication technologies [5]. In extrusion-based 3D printing, scaffolds are created by deposition of materials in a layer-by-layer fashion to create 3D structures. The major advantages of 3D printing are 1) the easily customizable external shape, 2) the high control over the internal architecture of the scaffold, and 3 ) the automated process to achieve consistent results. This allows the creation of scaffolds with a pre-determined pore size, porosity and pore interconnectivity, which is especially important in the areas of bone tissue engineering, where the pore size is a critical parameter that determines vascularization and osteointegration of a bone scaffold [6].

The development of inks for the extrusion based 3D printing of biomaterials (a type of biofabrication [7]) is one of the grand challenges to propel the technology forward. With a larger variety of biomaterial inks available, extrusion based biofabrication would become more attractive to a wider audience of researchers which might help to drive this technology towards clinical applications. Besides assessing the printability of newly created inks as outlined recently by Paxton et al. [8], great care should be taken throughout the entire development process regarding scalability and constant quality control of the manufactured inks [9]. It is therefore crucial to work with robust polymer synthesis methods and to use only materials and technologies which allow the manufacturing of large batches of ink. Here we describe the development and characterization of a potential new ink for the 3D printing of a bone layer of biphasic constructs such as osteochondral grafts. The ink is based on gellan gum methacrylate (GG-MA), hyaluronic acid methacrylate (HAMA) and hydroxyapatite (HAp) particles. Gellan gum (GG) based inks have recently been shown to have excellent printability [10] and both hyaluronic acid [11, 12] and hydroxyapatite [13-15] have been used in the past for bone tissue engineering applications. HAp can also be used to modify the rheological behaviour $[16,17]$ of GG-MA to enhance its printing properties. Methacrylation of the polymer components of the ink was chosen to facilitate crosslinking between two different materials 
(phases) of a biphasic constructs as well as to enable the creation of a strong interface between layers within the same phase.

Being of biological origin, gellan gum (as many other biopolymers) suffers from batch-to-batch variations and chemical modification of a particular batch of gellan gum can exacerbate this issue even further. To achieve consistent printing results, robustness of the synthetic procedure for gellan gum methacrylate is therefore crucial. Batch-to-batch variations in the degree of methacrylation influence not only the final mechanical properties of the printed graft but might as well influence the rheological properties of the ink [18]. In inks such as gelatine methacrylate (GelMa), methacrylate groups are introduced using methacrylic anhydride through the modification of the amine moieties present in the chain [19]. In other materials, such as hyaluronic acid, the methacrylic anhydride targets the primary alcohols [20]. Another method for methacrylation, which can target both carboxylic acids and primary alcohols [21], is the usage of glycidyl methacrylate (GMA) [22-24]. For this method, a stable $\mathrm{pH}$ of 8.5 during the reaction is needed in order to achieve high degrees of substitution within $24 \mathrm{~h}$. At pH 8.5, the transesterification reaction of GMA with the carboxylic acid moiety takes place, which proceeds faster and is therefore preferred over the slower ring-opening reaction on the epoxy group [25]. In the case of biopolymers such as gellan gum, a too high or too low $\mathrm{pH}$ further increases the risk of depolymerisation of the biopolymer chain [26]. Manual or automated adjustment of the $\mathrm{pH}$ to 8.5 has major drawbacks. Manual $\mathrm{pH}$ adjustment can lead to inconsistent degrees of methacrylation due to an unstable $\mathrm{pH}$ over time. Automated titrators negate the need for manual $\mathrm{pH}$ adjustments but measure the $\mathrm{pH}$ at a fixed position in the reaction vessel. This becomes problematic with larger reaction volumes for large lab scale synthesis, as the measured $\mathrm{pH}$ might no longer be representative of the entire vessel which causes the titrator to add the wrong amounts of acid or base into the reaction. Further, in the case of biopolymers such as gellan gum, the reactions are preferably carried out at low polymer concentrations to avoid high solution viscosities which would cause a reduction in mass transfer as well as an inconsistent $\mathrm{pH}$ throughout the reaction vessel.

The first goal of the presented work was therefore to develop a new method for the synthesis of large batches ( $5 \mathrm{~g}$ ) of GG-MA using buffered solutions to control the degree of methacrylation. GGMA synthesis is commonly carried out on the $1 \mathrm{~g}$ scale $[27,28]$ and occasionally on a $3 \mathrm{~g}$ scale [29], however, bigger batch sizes would enable the fabrication of larger ink volumes (in our case more than $100 \mathrm{ml}$ ) from a single batch of GG-MA, therefore reducing the potential for inconsistent printing results using cartridges from different ink production dates. The second goal of this work 
was to develop and evaluate methods to assess the reproducibility of the printing process. To assess how needle diameters affect the printing consistency, we compared different needle geometries and ink compositions through extrusion force measurements. These measurements can also be used to evaluate ink homogeneity within the cartridge and therefore the potential to achieve consistent printing results. Similar setups have been used in the past to assess the filter pressing effect and evaluate the injectability of bone cement pastes [30], but has to our knowledge not often been used to assess inks for 3D printing. Extrusion force measurements can not only show if air bubbles were introduced during the cartridge filling, but also reveal potential clogging due to aggregates or larger particles present in the ink. Particles such as hydroxyapatite[31] or laponite[32] are often added to inks to introduce biologically relevant materials or simply as rheological modifiers. Extrusion force measurements therefore enable us to choose the right needle size and shape for such particle containing inks to avoid clogging. Both air bubbles and clogging can lead to inconsistent printing results due to the altered flow during extrusion of the ink and are, although not often mentioned, common obstacles during scaffold fabrication using 3D printing and can jeopardize the mechanical integrity of larger scaffolds. Inks with a large content of solid particles are also not only prone to clogging but also to inhomogeneity within a cartridge due to insufficient mixing, particle aggregation or, in the case of lower viscosity inks, particle sedimentation. To further assess the ink homogeneity throughout the different locations of a printer cartridge, the ink compositions from different sections of the cartridge were evaluated with thermogravimetric analysis (TGA). To complete the evaluation of the printed constructs, the interfacial bond strength between two printed layers was analysed with the help of push-out tests. Finally, we investigated the viability and ability of mesenchymal stromal cell (MSCs) to adhere to the inks and analysed the surfaces of different ink compositions using scanning electron microscopy (SEM). 


\section{Materials and Methods}

\subsection{Materials}

All materials and reagents were purchased from Sigma-Aldrich, Invitrogen, Thermo Fisher Scientific or Merck Millipore and were used as received unless otherwise stated. The HAp particles were obtained from Acros Organics.

\subsection{Methods}

\subsubsection{GG-MA Synthesis}

GG-MA was synthesized in two ways: with manual pH control as previously described [23] or by using a buffered solution. For the synthesis with manual pH control, $1 \mathrm{~g}$ of low-acyl gellan gum (Kelco, USA) was dissolved in $100 \mathrm{ml}$ of Milli-Q water and heated to $90^{\circ} \mathrm{C}$ in a sealed Erlenmeyer flask. After the solution became transparent, indicating complete dissolution of the gellan gum, it was cooled down to room temperature under vigorous stirring. The $\mathrm{pH}$ was adjusted to 8.5 with 0.5 $\mathrm{M} \mathrm{NaOH}$ and a 20-fold molar excess of glycidylmethacrylate (GMA) was added. The $\mathrm{pH}$ of the reaction was manually maintained for 3 hours through drop wise addition of $0.1 \mathrm{M} \mathrm{NaOH}$. The reaction was allowed to proceed for a total of 24 hours after which the solution was precipitated in $50 \mathrm{ml}$ of cold acetone. The gel-like precipitate was placed in dialysis bags (12-14 kDa MWCO) and dialyzed against Milli-Q water for 7 days with water changes every hour during the first 6 hours and twice daily for the remaining 6 days. The product was finally freeze-dried and stored at $-20^{\circ} \mathrm{C}$.

For the buffer-mediated synthesis, gellan gum was dissolved in 2-amino-2-methyl-1,3-propanediol (AMPD) buffer (100 mM, pH 8.5) at 1\% w/v for batch sizes of up to $5 \mathrm{~g}$. GG was dissolved in the buffer in a sealed flask at room temperature and then heated up to $90^{\circ} \mathrm{C}$ under vigorous stirring until a clear solution was obtained. The solution was then cooled down to room temperature under vigorous stirring and GMA was added to the solution at various molar excesses ranging from 2.5 to 20 equivalents with respect to the carboxylic acid present in gellan gum. After 24 hours, the polymer was precipitated and purified in the same way as when synthesized with manual $\mathrm{pH}$ control. The degree of substitution (DS) was calculated by $1 \mathrm{H}-\mathrm{NMR}\left(\mathrm{D} 2 \mathrm{O}, 500 \mathrm{MHz}, 80^{\circ} \mathrm{C}\right)$ using the ratio of the integrals of the peaks from the methacrylate groups at $5.9 \mathrm{ppm}$ and $6.3 \mathrm{ppm}$ and the peak of the methyl group belonging to the rhamnose unit of gellan gum at $1.45 \mathrm{ppm}$. The structures of both GG and GG-MA are shown in Figure S1. 


\subsubsection{HAMA Synthesis}

HAMA was synthesized as described previously in the literature [33]. Briefly, 1\% low molecular weight HA (280 kDa) was dissolved in ultrapure water overnight. The next day, the solution was placed in an ice bath and the $\mathrm{pH}$ was adjusted to 8.0 with $\mathrm{NaOH}$. A 20-fold molar excess (per disaccharide unit) of methacrylic anhydride was then added under vigorous stirring. During the first three hours, the $\mathrm{pH}$ was maintained at $\mathrm{pH} 8.0$ by adding $5 \mathrm{M} \mathrm{NaOH}$ after which the reaction was allowed to proceed for $24 \mathrm{~h}$ at $4^{\circ} \mathrm{C}$. Unlike GMA, methacrylic anhydride does not react via two different reaction mechanisms depending on the $\mathrm{pH}$ which is why a constant $\mathrm{pH}$ over $24 \mathrm{~h}$ is less critical. After $24 \mathrm{~h}$, HAMA was precipitated by dripping the solution into an excess of ice-cold ethanol. The precipitate was then collected by vacuum filtration. After redissolving the precipitated HAMA in ultrapure water, it was dialyzed for three days against ultrapure water (Spectra/Por 5, MWCO 12-14 kDa). The water was changed every 24 hours. The product was freeze-dried and stored at $-20^{\circ} \mathrm{C}$ before use. NMR spectroscopy $(\mathrm{D} 2 \mathrm{O}, 500 \mathrm{MHz})$ revealed successful methacrylation of the hyaluronan. The degree of substitution (DS) was calculated by the ratio of the integrals of the vinyl protons at $5.6 \mathrm{ppm}$ and $6.1 \mathrm{ppm}$ to the protons from the methyl groups of both, HA and the methacryloyl residues at $1.9 \mathrm{ppm}$. The calculated degree of substitution for the batches utilized in this work ranged from 31.2-34.6\%.

\subsubsection{Hydroxyapatite Particle Analysis}

The size distribution of the HAp particles was evaluated by means of light scattering using a Malvern MasterSizer 2000. The particles were suspended in degassed water using a sonicator probe and analyzed immediately to avoid particle sedimentation. The size distribution of the HAp particles is shown in Figure S2.

\subsubsection{Size Exclusion Chromatography}

Size exclusion chromatography (SEC) was performed to determine the influence of the synthesis and cryomilling on the molecular weight of GG and HA and their derivatives. Each sample was solubilized in $100 \mathrm{mM} \mathrm{NaNO}_{3}$ containing $5 \mathrm{mM}$ of the eluent $\mathrm{NaN}_{3}$. Solubilization took place over night at $37^{\circ} \mathrm{C}$ for the $\mathrm{HA}$ samples and $a 0^{\circ} \mathrm{C}$ for the GG sample to prepare $0.1 \%$ solutions for analysis. Before injection, the samples were passed through a $0.45 \mu \mathrm{m}$ filter. The HPLC setup used for the SEC measurements included a binary pump, degasser, thermostated column compartment and an auto sampler, all from HP (Series 1100, Hewlett Packard, USA). A pre-column (Viscotek AGuard Col. $50 \mathrm{x}$ $6.0 \mathrm{~mm}$, Malvern Instruments Ltd, United Kingdom) was used together with a A5000 column (Viscotek, $300 \times 7.8$ mm, Malvern Instruments Ltd, UK) and a suprema 30000 column (10 $\mu \mathrm{m}, 8 \times 300$ 
$\mathrm{mm}$, PSS Polymer Standards Service GmbH, Germany). The temperature of the columns was kept at $35{ }^{\circ} \mathrm{C}$ with

a flow rate of $1 \mathrm{~mL} / \mathrm{min}$ and an injection volume of $50 \mu \mathrm{L}$. The elution was recorded using a refractive index detector (Series 1200, Agilent Technologies, Switzerland). The average molecular weights were calculated based on the measured standard curve with the ChemStation software (ChemStation for LC 3D systems, Rev B.04.02 SP1) and the add-on Cirrus GPC/SEC software (version 3.4.1) from Agilent.

\subsubsection{Ink Preparation and Cartridge Loading}

To facilitate dissolution of the lyophilized polymers (retrieved as white sponges), GG-MA and HAMA were milled using a cryomill (Retsch, Switzerland, $25 \mathrm{ml}$ grinding jar, $15 \mathrm{~mm} \varnothing$ grinding balls). GG-MA and HAMA were placed into a pre-cooled grinding jar at a weight ratio of 2.67:1 and milled for 2 minutes at $-196^{\circ} \mathrm{C}$ at a frequency of $30 \mathrm{~Hz}$. A fine powder was obtained which was stored at $-20^{\circ} \mathrm{C}$ until further use. For the mixing of the materials, a planetary centrifugal mixer (ARE-250CE, ThinkyMixer, Japan) was utilized. MilliQ water was placed in a container and GG-MA/HAMA powder was added to achieve a final concentration of $4 \% \mathrm{w} / \mathrm{v}$ GG-MA and $1.5 \% \mathrm{w} / \mathrm{v}$ HAMA (from here on named "ink"). Different amounts (\% w/w) of hydroxyapatite particles were then added to the container and mixed for $20 \mathrm{~min}$ at $2000 \mathrm{rpm}$. A warm paste was obtained from the mixing process which was further stirred with a spatula until the paste cooled down to room temperature. The ink was stored in the mixing container at $4^{\circ} \mathrm{C}$ until utilized for printing. Before loading the ink into the printer cartridge, the photoinitiator Irgacure 2959 (Ciba, Switzerland) was added to obtain a final concentration of $0.05 \%(\mathrm{w} / \mathrm{v})$. The ink containing Irgacure 2959 was filled into the printing cartridges ( $3 \mathrm{cc}$, Nordson EFD, Switzerland) using an Omnifix syringe (Braun Medical Inc., USA) and a female luer-lock connector piece. Care was taken not to trap air during the loading procedure. In case air was trapped, centrifugation of the loaded cartridges was performed at $500 \mathrm{rpm}$ (Z400K, Hermle, Germany).

\subsubsection{Rheological Measurements}

The influence of the GG-MA synthesis procedure, hydroxyapatite content as well as polymer processing on the rheological properties of the ink was assessed using an MCR 301 rheometer (Anton Paar, Switzerland). The rheometer was equipped with a Peltier element, a thermostatic hood for temperature control and a UV Source (Omnicure Series 1000, 320-500 nm wavelength, 9.55 $\mathrm{mW} / \mathrm{cm}^{2}$ ) (Lumen Dynamics, Canada) for UV-crosslinking. The samples were covered with a low viscosity silicon oil to avoid evaporation of water from the samples and all measurements were performed at $25^{\circ} \mathrm{C}$. A profiled parallel-plate ( $20 \mathrm{~mm}$ diameter, $0.5 \mathrm{~mm}$ profile) setup was used to 
avoid wall-slip. The flow properties of the inks were evaluated through a shear rate sweep from 0.001 to $1000 \mathrm{~s}^{-1}$. The shear recovery experiments were performed under oscillation by measuring the storage $\left(G^{\prime}\right)$ and the loss modulus $\left(G^{\prime \prime}\right)$ at an angular frequency of $5 \mathrm{rad} / \mathrm{s}$ and a low strain of $0.01 \%$ which was found to be within the linear viscoelastic range (LVR). The strain was then increased to $150 \%$ at the same frequency for 150 seconds, before returning back to $0.01 \%$ strain for 600 seconds. For the UV-crosslinking of the ink, $G^{\prime}$ and $G^{\prime \prime}$ were recorded for 60 seconds before illuminating the samples with UV-light for 300 seconds while measuring the evolution of $\mathrm{G}^{\prime}$ and $\mathrm{G}^{\prime \prime}$. To assess the relaxation behaviour of the ink, the normal force upon compression of the sample down to a $0.5 \mathrm{~mm}$ gap was recorded over the course of 60 minutes. All rheological curves represent the average of two measurements of separate samples.

\subsubsection{Extrusion Force Measurements}

Extrusion force measurements were performed on a Texture Analyzer XT Plus (Stable Micro Systems, United Kingdom) equipped with a custom-made extrusion setup (link to the CAD files can be found in the supplementary information). Printer cartridges were filled with inks containing different amounts of HAp and placed into the holder. Total ink volume in the cartridges differed between the different samples. The plunger of the cartridges was pushed down at a constant displacement rate of $0.25 \mathrm{~mm} / \mathrm{s}$ until all ink was pushed out of the cartridge. The force was assessed using a load cell (max. load 5 kg, Stable Micro Systems).

\subsubsection{Thermogravimetric Analysis}

TGA measurements were performed to assess the hydroxyapatite content in the ink from different parts of a $3 c c$ printer cartridge which was prepared according to section 2.2.5. Samples were measured on a TGA/SDTA851e (Mettler Toledo, Switzerland) in air. 3cc printer cartridges were filled with ink and extruded using the printer at a pressure $95 \mathrm{kPa}$. Samples were taken from different fractions of the extrudate. A fraction was defined as $1 / 6$ of the total ink volume of the cartridge. The fractions were then dried in a vacuum oven for 2 hours at $60^{\circ} \mathrm{C}$ to remove residual free water and subsequently placed in alumina crucibles. They were measured in the TGA apparatus by heating the sample from $50^{\circ} \mathrm{C}$ to $900^{\circ} \mathrm{C}$ with a temperature ramp of $5^{\circ} \mathrm{C} / \mathrm{min}$. The weight was recorded as percentage of the initial sample weight at $50^{\circ} \mathrm{C}$.

\subsubsection{Printing Characterization}

All printing was performed on a BioFactory bioprinter (regenHU, Switzerland). Designs for the printing were created using the BioCAD software (regenHU, Switzerland) or with Slic3r (http://slic3r.org/) in combination with a custom written MATLAB postprocessor. All samples were 
printed at room temperature and crosslinking was performed using a built-in UV-Pen ( $365 \mathrm{~nm}, 6.09$ $\left.\mathrm{mW} / \mathrm{cm}^{2}\right)$.

To assess the influence of printing speed on the strand size of ink from different compartments of the cartridge, single lines were printed at printing speeds between $50-1600 \mathrm{~mm} / \mathrm{min}$ and their widths as well as heights were evaluated using ImageJ. This was performed for inks with $25 \%$ and $30 \%$ HAp content extruded with $0.61 \mathrm{~mm}$ conical and a $0.41 \mathrm{~mm}$ straight steel needle (Nordsen, USA). The extrusion pressures were chosen so that a continuous flow of ink from the cartridge was obtained. To show the suitability of the developed ink for biphasic constructs (e.g. osteochondral), ink with $0 \%$ HAp content was used for the top phase and ink with $25 \%$ HAp content was used for the bottom phase of the biphasic construct and both inks were printed on top of each other using a 0.41 $\mathrm{mm}$ straight steel needle. Inks contained $0.05 \% \mathrm{w} / \mathrm{v}$ Irgacure 2959. Passage 4 bovine chondrocytes were stained with CellTracker Blue (4-chloromethyl umbelliferone, $10 \mu \mathrm{M}$ ) for 30 minutes, washed with serum-free media and gently mixed into the $0 \% \mathrm{HAp}$ ink at $10 \cdot 10^{6}$ cells $/ \mathrm{ml}$ using a spatula. Printing of the HAp containing ink layer was performed at a pressure of $103 \mathrm{kPa}$ and a speed of 215 $\mathrm{mm} / \mathrm{min}$. A total of 4 layers was printed. 2 layers with $0 \%$ HAp ink were then printed on top with a pressure of $25 \mathrm{kPa}$ and a speed of $400 \mathrm{~mm} / \mathrm{min}$. UV-crosslinking was performed for 2 min after every printed layer and for an additional 5 minutes after the end of the top layer.

\subsubsection{Interfacial Bond Strength}

To assess the interfacial bond strength between two printed layers, an outer ring of $25 \%$ HAp content ink (representing the first printed layer) with an inner diameter of $4 \mathrm{~mm}$ was printed using a $0.61 \mathrm{~mm}$ conical needle, a pressure of $60 \mathrm{kPa}$ and a printing speed of $400 \mathrm{~mm} / \mathrm{min}$. This first layer was then crosslinked for either 2,4 or 8 min which simulates the curing process before deposition of the next layer. The ring was then filled manually (representing the second printed layer) with either $25 \%$ HAp ink, 0\% HAp ink, 1\% HAMA or 30\% Pluronic-dimethacrylate mixed with $5 \%$ chondroitin sulfate-methacrylate (PF-CSMA) to assess the interfacial bond strength between different inks of a potential biphasic construct. To clarify the different combinations, we illustrated them in Figure 1. 


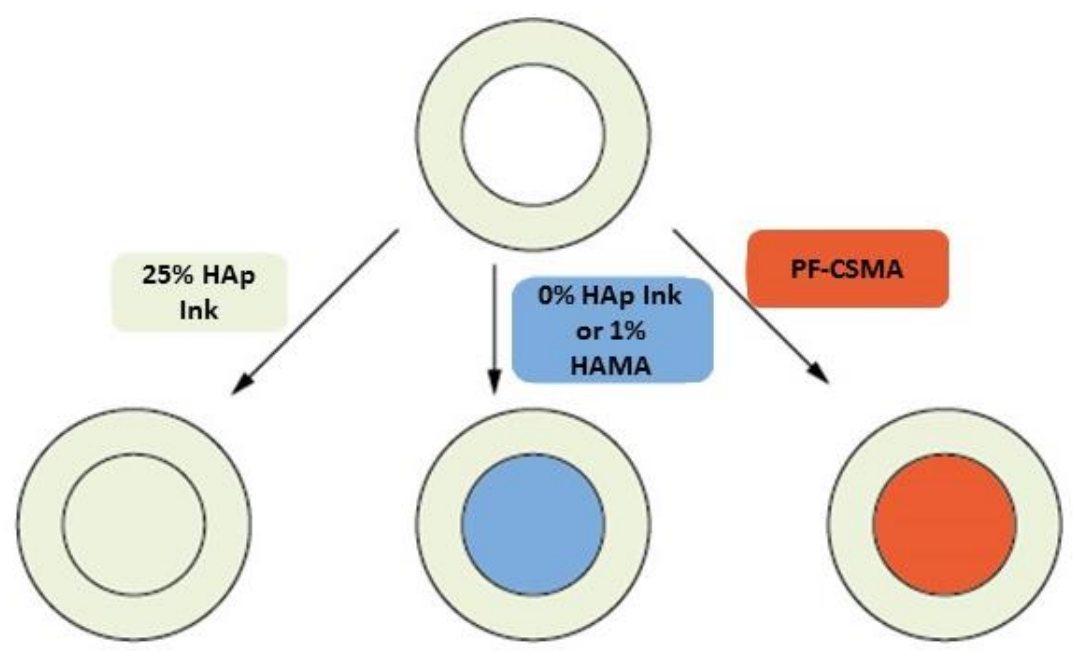

Figure 1: Different outer-inner layer configurations for the push-out tests

The interface between the two layers was then illuminated with UV-light with the UV source moving along the interface at a speed of $7.85 \mathrm{~mm} / \mathrm{min}$ for a total of $2 \mathrm{~min}$. After another $5 \mathrm{~min}$ of UVexposure in the center of the second layer (representing the final crosslinking step after printing), the samples were placed in a sample holder and the push-out test was performed at $0.5 \mathrm{~mm} / \mathrm{s}$ on a Texture Analyzer XT Plus (Stable Micro Sytems, United Kingdom). The sample holder consisted of a steel ring with an inner diameter of $5 \mathrm{~mm}$ and the probe head had a diameter of $3 \mathrm{~mm}$. The force curve was recorded and the maximum force was divided by the interfacial area between the two layers to obtain the interfacial bond strength. Each interface area was calculated separately by quantifying the inner radius of the inner circle material with light microscope and carefully measuring the height of the printed sample using a calliper.

\subsubsection{Cell Adhesion and Viability}

The MSCs used in the adhesion and viability study were obtained from trabecular bone samples that were retrieved during surgical hip replacement of an otherwise healthy patient (male, 42 years) after having received informed consent. Approval of the protocol was obtained from the ethical board of the Kantonsspital St. Gallen, Switzerland (ethical committee approval number EKSG08/014/1B). The samples were incubated overnight at $4^{\circ} \mathrm{C}$ in isolation medium ( $25 \mathrm{mM} \mathrm{HEPES}, 128.5 \mathrm{mM} \mathrm{NaCl}, 5.4$

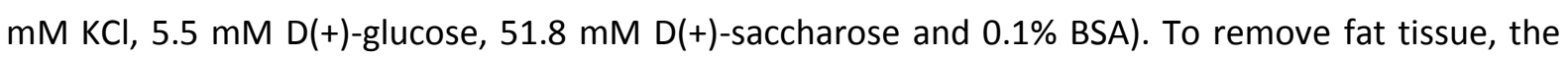
sample was centrifuged at $110 \mathrm{~g}$ for $15 \mathrm{~min}$ at $4^{\circ} \mathrm{C}$ and the remaining pieces of trabecular bone were rinsed with isolation media several times. The solution was collected and filtered through a $200 \mu \mathrm{m}$ cell strainer. The solution was centrifuged and the obtained cell pellet was resuspended in proliferation media (DMEM 31885, 10\% FBS, 1\% Pen-Strep and $5 \mathrm{ng} / \mathrm{ml} \mathrm{FGF-2).} \mathrm{Passage} 3$ MSCs were used in the adhesion study. 
The samples for the adhesion study were prepared in a rheometer setup by UV-crosslinking resulting in discs with a diameter of $20 \mathrm{~mm}$ and a thickness of $0.5 \mathrm{~mm}$. Discs with a diameter of $6 \mathrm{~mm}$ were punched out using a biopsy punch and placed in sterile $100 \mathrm{mM} \mathrm{CaCl}_{2}$ overnight. $70 \%$ ethanol was then put in the wells for 10 minutes to disinfect the surface. This was followed by two washing steps with proliferation media. The discs were left to incubate in proliferation media for 1 hour. The MSCs were then seeded on the ink disc containing different amounts of HAp at $40^{\prime} 000$ cells/cm2 and cell adhesion was assessed after 3 days using a phalloidin-rhodamine/Hoechst staining. For the staining, the media was removed and the samples washed once with PBS and subsequently fixed with $4 \%$ formaldehyde in PBS for 25 min. After two more washes with PBS, the cells were permeabilized for 15 min using $0.1 \%$ Triton-X100 + 1\% BSA. The solution was removed from the samples and they were rinsed 3 times with PBS. Phalloidin-rhodamine at a final concentration of $0.13 \mu \mathrm{M}$ in serum-free media was then added to the samples for $30 \mathrm{~min}$, after which Hoechst ( $10 \mu \mathrm{g} / \mathrm{ml}$ final concentration) was added and incubated with the samples for another $30 \mathrm{~min}$. The samples were washed 3 times for 1 minute with PBS and imaged with a fluorescent microscope (Zeiss Axio Observer, Zeiss, Switzerland).

For the viability measurements using live/dead staining, the seeded cells were washed once with media without FBS and subsequently stained with $1 \mathrm{ug} / \mathrm{ml}$ Calcein AM (1uM) and $0.66 \mathrm{ug} / \mathrm{ml}$ Propidium lodide (1uM) for $15 \mathrm{~min}$. This was followed by a single wash with cell culture media and the cells were then imaged with a fluorescent microscope.

To analyse the cell viability using the MTS assay, MTS stock solution was prepared according to the manufacturer's instructions (CellTiter $96^{\circledR}$ AQueous One Solution, Promega). MTS stock solution was then diluted to $0.04 \%$ in cell culture medium (150ul medium with 30 ul MTS stock solution). Samples were incubated for $1 \mathrm{~h}$ and afterwards $150 \mathrm{ul}$ of solution was placed in 48 well-plate and read out on a microplate reader (Synergy H1, BioTek) at $490 \mathrm{~nm}$. Cell culture media was utilized as blanks and cells seeded on tissue culture plastic (TCPS) were used as positive and cells treated with $70 \%$ ethanol for $30 \mathrm{~min}$ were used as negative controls.

\subsubsection{Scanning Electron Microscopy}

For scanning electron microscopy (SEM), discs were created as described earlier using different amounts of HAp. To investigate if there is hydroxyapatite crystal formation on the gellan gum containing ink as previously reported elsewhere [34], the discs were placed in $\mathrm{CaCl}_{2}$ overnight before being exposed to cell culture media (without FBS) for 7 days at $37^{\circ} \mathrm{C}$ in an incubator $\left(5 \% \mathrm{CO}_{2}\right)$. Afterwards they were frozen at $-80^{\circ} \mathrm{C}$ and placed into a lyophilizer for freeze-drying. The freeze-dried 
discs were then coated with a $5 \mathrm{~nm}$ layer of platinum and subsequently analyzed in a Zeiss Gemini 1530 FEG SEM at $5 \mathrm{kV}$, at room temperature.

\section{Results}

\subsection{Buffer Mediated GG-MA Synthesis}

Robust synthesis methods that involve a minimal number of manual steps are essential for the reproducible production of large polymer batches to develop inks for 3D printing applications. As 3D printing often requires large volumes of ink (several $\mathrm{mL}$ ) for large scaffold production, up-scaling of the synthesis is required. By utilizing 2-amino-2-methyl-1,3-propanediol (AMPD) as a buffer, we were able to eliminate the need to manually balance the $\mathrm{pH}$ at 8.5. Over the course of 3 hours, the $\mathrm{pH}$ of the reaction only decreased from 8.5 to 8.33 and after 24 hours we found a final $\mathrm{pH}$ of 7.91 . Furthermore, the buffer mediated synthesis achieved a 2.5 times higher degree of substitution than the conventional synthesis method (Figure 2a, red square) when a 20-fold molar excess of GMA was used in the reaction. The methacrylation of GG using GMA followed a linear trend up to 15 -fold molar excess and batch-to-batch differences were small (1.76 $\pm 0.14 \%$ for the 5 -fold, $1.72 \pm 0.20 \%$ for the 5.6 -fold and $3.14 \pm 0.12 \%$ for the 10 -fold excess, respectively). An increase to a 20 -fold excess does not increase the DS, indicating that there is a kinetic limit of how many functional groups on GG can be modified within $24 \mathrm{~h}$, independent on the excess of GMA. 

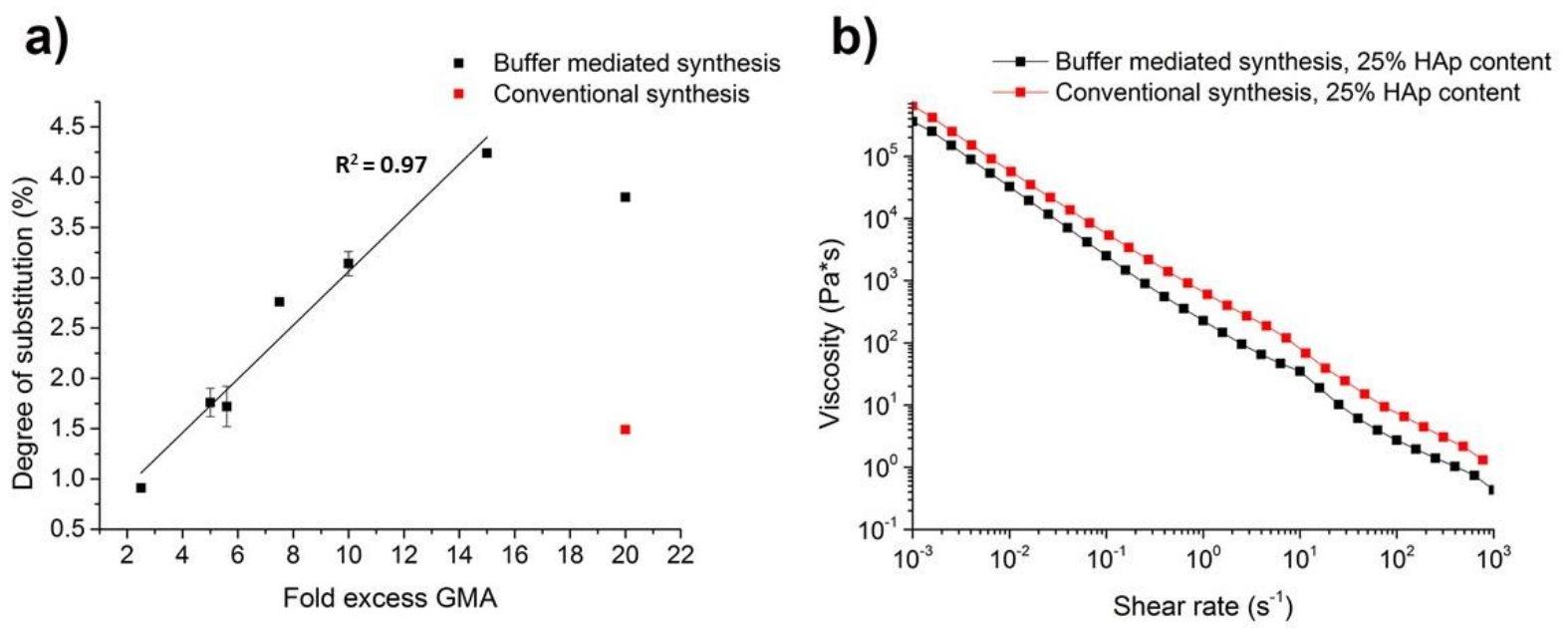

Figure 2: a) Obtained degrees of substitution (DS) in buffer mediated GG-MA synthesis with varying fold-excess of GMA. A 2.5 higher DS was achieved with buffer mediated synthesis (black squares) compared to conventional synthesis (red square) at 20-fold molar excess. b) Influence of the synthesis method on the flow behaviour of inks with $25 \% \mathrm{HAp}$. The buffer mediated synthesis was performed with 5 -fold excess GMA.

To evaluate the influence of the synthesis method on the properties of the ink, GG-MA produced by the conventional synthesis method (20-fold GMA excess) was compared with buffer synthesized GGMA (5.6-fold GMA excess) in all experiments as those had the same degree of substitution. Rheological measurements were performed to assess the flow behaviour of inks produced with either synthesis method. As seen in Figure 2b, the viscosity of an ink with 25\% HAp content was decreased at all shear rates when the buffer mediated synthesized GG-MA was used. The same trend was found for ink containing 30\% HAp (data not shown), indicating that the synthesis method for GG-MA indeed has an influence on the rheological behaviour of the produced HAp containing inks.

\subsection{Influence of Polymer Processing}

High molecular weight biopolymers are usually obtained from lyophilization as low-density, spongelike materials. This complicates the creation of solutions with a high polymer content as complete hydration of these sponges with small amounts of water often proves difficult in our experience. We therefore decided to mechanically grind the obtained GG-MA and HAMA sponges into a powder using a cryomill to facilitate ink production. When inks with $30 \%$ HAp content and cryomilled polymers were rheologically assessed, we found differences in the viscosity of up to one order of magnitude (11.3 $\mathrm{kPa} \cdot \mathrm{s}$ at versus $113 \mathrm{kPa} \cdot \mathrm{s}$ at a shear rate or $\left.0.1 \mathrm{~s}^{-1}\right)$ compared to the untreated polymers as shown in Figure 3. 


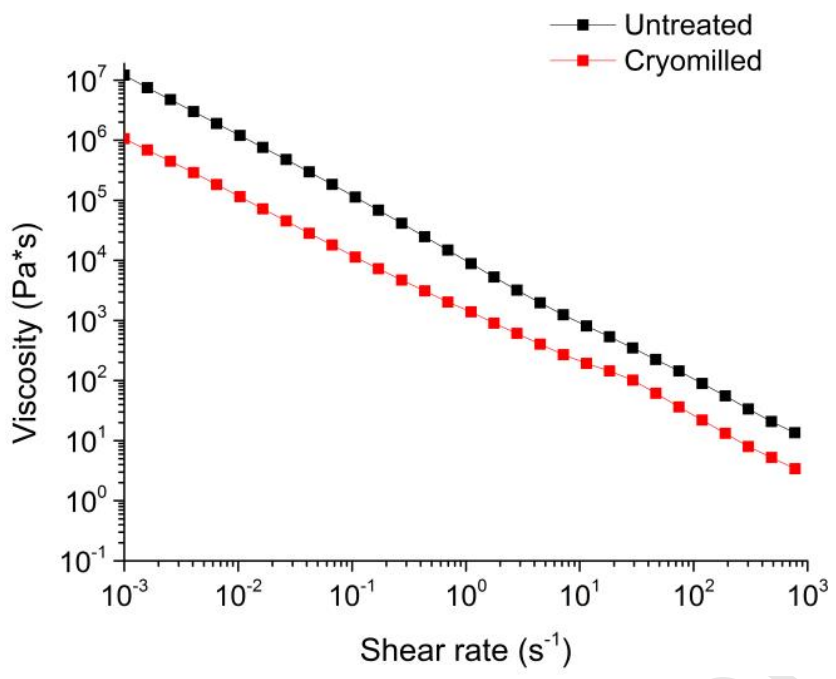

Figure 3: Influence of the cryomilling process on the rheological behaviour of an ink containing $30 \%$ HAp. In case of the untreated polymers, mixing was performed at $50^{\circ} \mathrm{C}$ in a sealed vessel under constant stirring. The GG-MA in the ink was synthesized using the conventional synthesis method.

To evaluate if this difference is caused by a reduction in the biopolymers' molecular weights, SEC measurements were performed on the underivatized HA and GG, their methacrylated versions HAMA and GG-MA and the cryomilled HAMA and GG-MA. As shown in Table S1, Mw of HA increased with the addition of the methacrylate groups to the polymer from 516 to $534 \mathrm{kDa}$. The mass increase of $18 \mathrm{kDa}$ correlates well with the added methacrylate groups at the obtained degrees of substitution. Upon cryomilling, the HAMA chains become fragmented which leads to a reduction of the $\mathrm{Mw}$ to $118 \mathrm{kDa}$ and an increase in the polydispersity index (PDI) from 2.63 to 4.01 . In case of GG, the addition of methacrylates reduced the $\mathrm{Mw}$ from 4,587 to 4,394 kDa which is much higher than expected from previous reports [35]. Cryomilling almost halved the Mw of GG-MA to 2,542 kDa, indicating the mechanical disruption of the chains. Both the scission of the HAMA and GG-MA chains during cryomilling are in agreement with a reduction of the viscosities of the inks (Figure 3).

\subsection{Influence of HAp content on flow behaviour and UV-crosslinking}

Particles in suspensions are known to introduce the possibility of shear thickening due to shear induced particle aggregate formation [36]. This effect would lead to undesirable clogging of the needle in a 3D printing application. To investigate the potential of the inks to undergo shear thickening, we assessed the flow behaviour of inks with 25\% and 30\% HAp content (Figure 4a) using rotational rheology. The two concentrations were chosen based on maximizing the amount of HAp for high printing fidelity while still being able to print the material without clogging the nozzle. For comparison, the flow curve of a $0 \%$ HAp content ink is depicted. Shear thickening was not observed 
at any of the shear rates investigated for the particle containing inks. Fitting of the flow curve data using the Herschel-Bulkley equation [37] revealed a yield point of $357 \pm 23 \mathrm{~Pa}$ and $516 \pm 55 \mathrm{~Pa}$ for the ink with $25 \%$ and $30 \% \mathrm{HAp}$ content, respectively. When the inks with $25 \%$ and $30 \% \mathrm{HAp}$ content were mixed with the photoinitiator Irgacure 2959 and exposed to UV radiation (320-500 nm bandpass filter), crosslinking occurred and after 5 minutes the inks with $25 \%$ and $30 \%$ HAp content reached storage moduli of 50.5 and $82.4 \mathrm{kPa}$ (Figure 4b), respectively. Using equation Eq. (1) we can calculate the shear modulus.

$\mathrm{G}=\sqrt{\left(G^{\prime}\right)^{2}+\left(G^{\prime \prime}\right)^{2}}$

For the inks with $25 \%$ and $30 \%$ HAp content the calculated shear moduli were 50.6 and $82.9 \mathrm{kPa}$, respectively.

For an ink for extrusion printing, quick recovery after a high shear event such as extrusion through a needle is crucial. If the shear recovery of an ink is slow, it will continue to flow after deposition onto a substrate diminishing the resolution that can be achieved. Shear recovery experiments were therefore performed on the $25 \%$ ink to see if structural changes occur during shear and if the ink recovers quickly after the shear event. After the first high shear event we found that $G^{\prime}$ recovered to $45 \%$ of its initial value within $30 \mathrm{~s}$ as shown in Figure 4c. After the second shear event, $\mathrm{G}^{\prime}$ recovered to only $30 \%$. Within $30 \mathrm{~s}$ after the third shear event, $27 \%$ of the initial $\mathrm{G}^{\prime}$ value was obtained. These results indicate that repeated shear events induce structural changes in the ink.
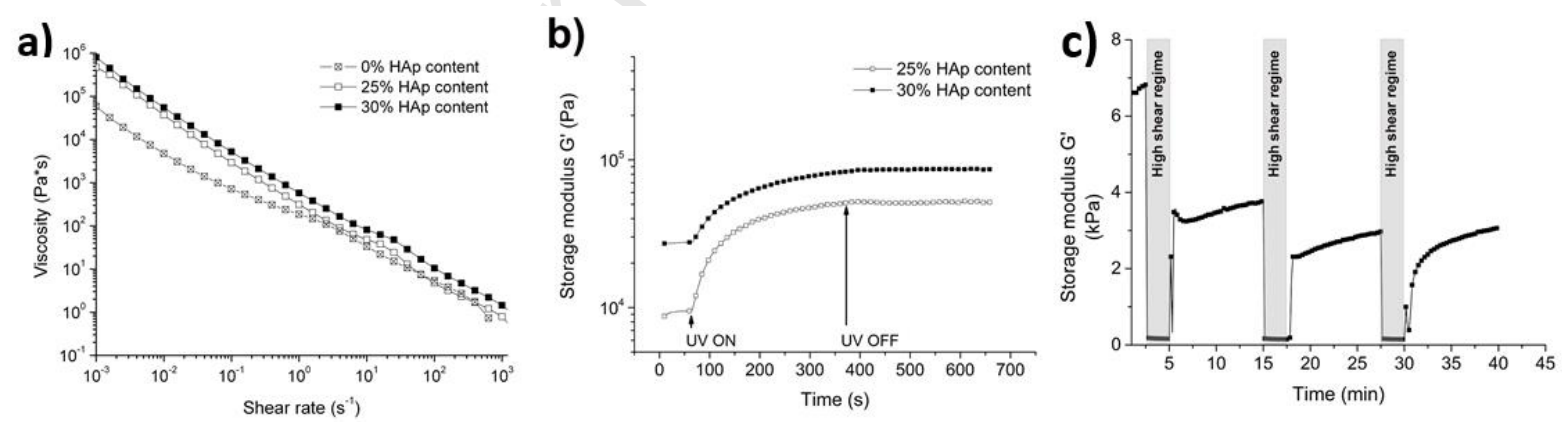

Figure 4: Flow behaviour of HAp containing inks compared to a $0 \%$ HAp ink (a) and changes in the storage modulus $G^{\prime}$ upon UV exposure (b) of inks with $25 \%$ and $30 \%$ HAp content. (c) Behaviour of the storage modulus $\mathrm{G}^{\prime}$ of an ink with $25 \%$ HAp content upon repeated exposure to high shear. 


\subsection{Extrusion Force Measurements}

While rheological analysis of inks for 3D printing can deliver information about their flow behaviour, it is not able to predict the extrusion performance through differently sized needle geometries. We therefore developed a setup which can be loaded with the same cartridges that are used in the printing process (Figure 5a).

a)

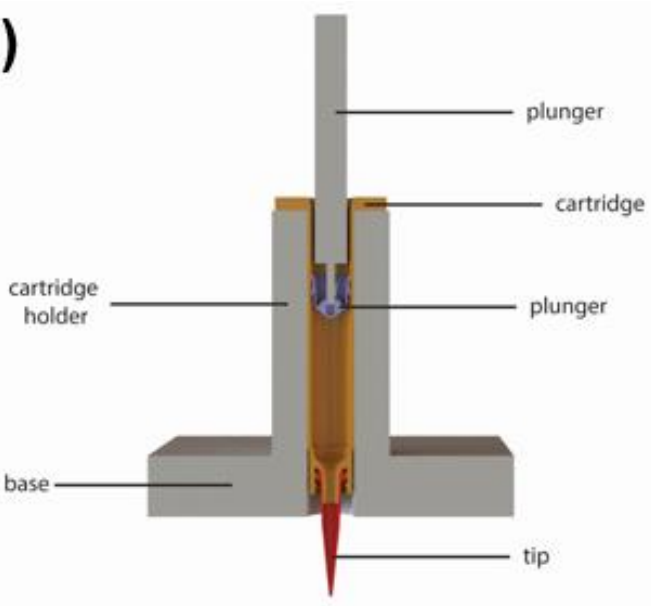

c)

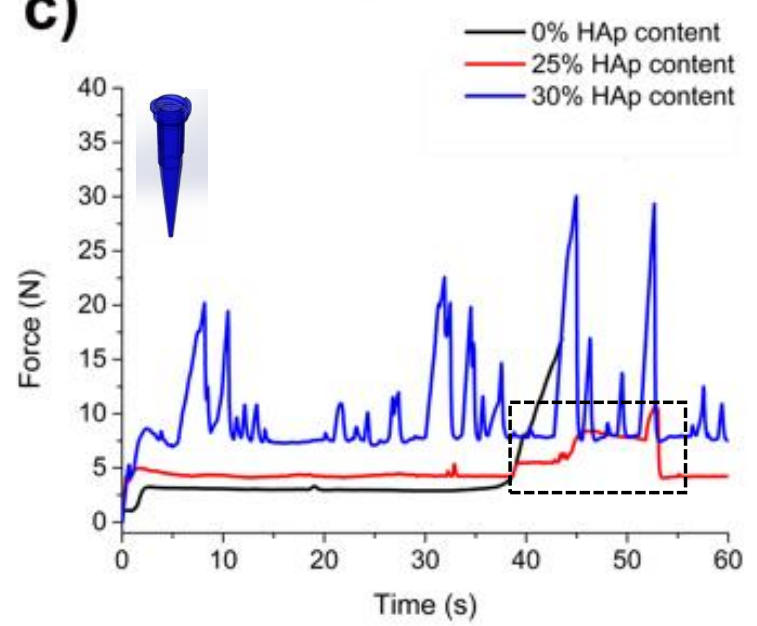

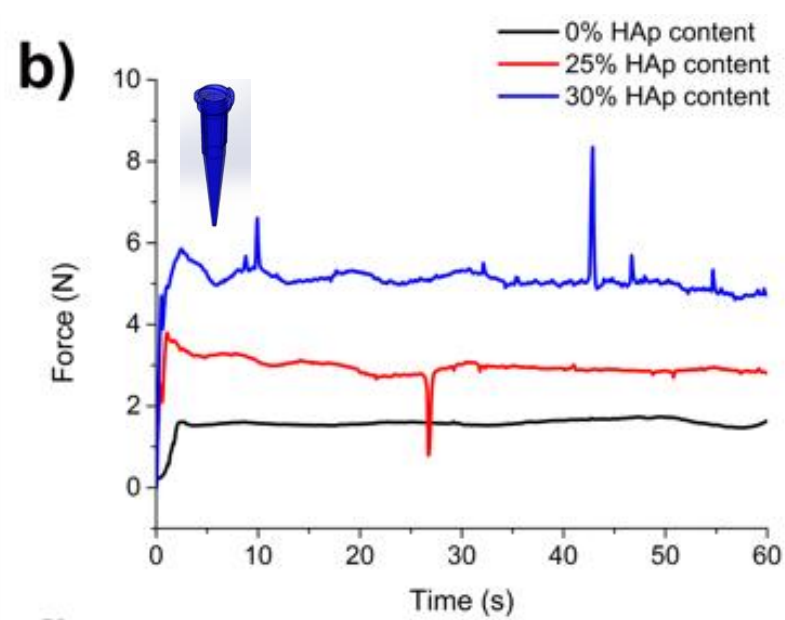

d)

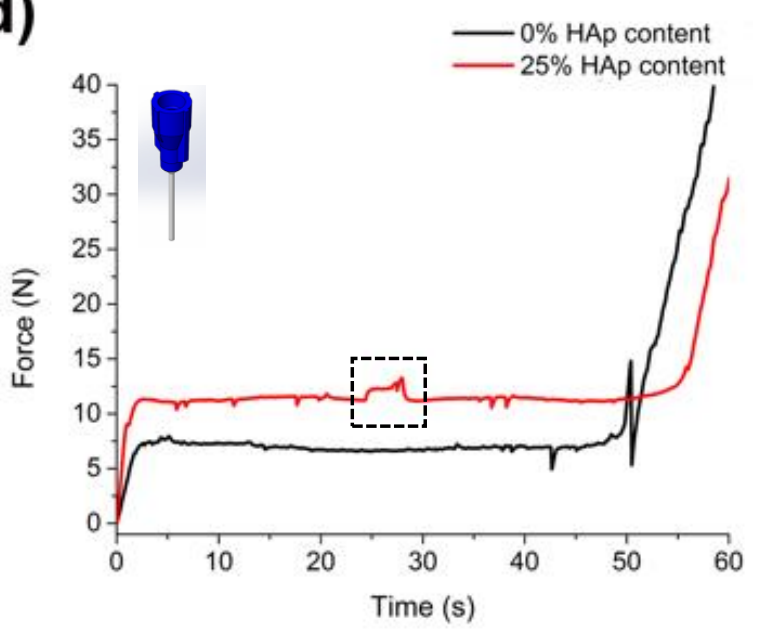

Figure 5: a) The extrusion force measurement setup consists of a plunger connected to the texture analyzer, the cartridge holder and the printer cartridge with the piston and ink. Inks with different HAp contents were extruded through. b) $0.61 \mathrm{~mm}$ conical needle, c) $0.41 \mathrm{~mm}$ conical needle and d) $0.41 \mathrm{~mm}$ straight steel needle. The $30 \%$ HAp sample is not shown as it was impossible to be extruded though a $0.41 \mathrm{~mm}$ straight steel needle. The insert in the subfigures $b, c$ and $d$ shows the needle type utilized for the extrusion force experiment. Dashed boxes in subfigures $\mathrm{c}$ and $\mathrm{d}$ highlight broad peaks from an unknown origin.

With this setup we were able to measure the extrusion force required for different needle geometries and ink compositions. Furthermore, with this method it is possible to assess the quality of the ink mixing process and cartridge filling. Inhomogeneous inks are revealed by a constant 
fluctuation of the extrusion force with aggregates being indicated by a positive peak whereas, on the other hand, air introduced during the cartridge filling procedure is indicated by a negative peak in the extrusion force. When inks with 0, 25 and 30\% HAp content were extruded from the printer cartridge equipped with a $0.61 \mathrm{~mm}$ conical printing needle (Figure $5 \mathrm{~b}$ ), the extrusion force was the highest for the ink with $30 \%$ HAp content. The extrusion force decreased with decreasing amounts of HAp particles in the ink. This was expected as the viscosities of the inks also decrease with decreasing HAp content (Figure 4a). The ink with 30\% HAp content displayed positive peaks in the recorded force curve indicating the presence of larger aggregates in the ink that cause short flow instabilities which hinder continuous printing. In the ink with 25\% HAp content, no positive peaks were observed. However, we observed a negative peak which represents an air bubble trapped during cartridge filling. The same ink without any HAp (0\% HAp content) did not display any peaks. Compared to the $0.61 \mathrm{~mm}$ conical needle, extrusion forces for all inks increased when extruded from a cartridge equipped with a smaller conical needle of $0.41 \mathrm{~mm}$. In the case of the ink with $30 \% \mathrm{HAp}$ content, positive peaks frequently occurred as shown in Figure $5 \mathrm{c}$. This means that aggregates with sizes in range of the inner needle diameter are present in the ink. The 30\% HAp ink is therefore unsuitable for printing with a $0.41 \mathrm{~mm}$ conical needle. In the case of the ink with $25 \%$ HAp content, no bubbles or peaks were observed apart from a very broad peak between 38-53 sec (dashed box in Figure 5c). A broad peak of similar shape was observed for the $25 \%$ HAp ink extruded through a straight $0.41 \mathrm{~mm}$ steel needle (dashed box in Figure $5 \mathrm{~d}$ ) and it is currently unclear if it is caused by aggregates or an artefact from the measurement setup. In the case of the $0 \%$ HAp ink, extrusion through a $0.41 \mathrm{~mm}$ conical needle was possible without any clogging and no bubbles were introduced during the cartridge filling process. The sharp increase in the extrusion force indicates that all the ink contained in the cartridge was extruded and the plunger hit the bottom of the cartridge. This happened earlier for the $0 \%$ HAp inks as smaller volumes of $0 \%$ HAp ink was utilized compared to the $25 \%$ and $30 \%$ HAp inks. To see if there are differences in the extrusion behaviour of inks between conically shaped and straight needles, they were extruded through a $0.41 \mathrm{~mm}$ straight steel needle (Figure $5 \mathrm{~d}$ ). Due to the fact that the $30 \%$ HAp ink was already considered unsuitable to be printed using a conical $0.41 \mathrm{~mm}$ needle, it was excluded from the experiment. Compared to the conical needle, both the inks with $0 \%$ and the $25 \%$ HAp content required a higher force to be extruded through the straight needle. The $0 \%$ HAp content ink did not show any clogging behaviour and the same was true for the $25 \%$. However, there was a broad peak between $24-28 \mathrm{sec}$ with a similar shape as the broad peak in Figure $5 c$ but with a smaller peak size compared to the overall extrusion force. We also tested if the clogging observed during the extrusion is based on pre-existing agglomerates or originates from agglomerates which formed during the ink preparation process. To 
do so, we sieved the HAp particles through a sieve with a $40 \mu \mathrm{m}$ mesh. As is shown in Figure S3a, sieving of the particles before the ink preparation lowered the extrusion force for the inks but did not prevent the aggregates altogether, indicated by the frequent appearance of positive peaks during the measurement. However, sieving of the particles enabled to create inks with $30 \% \mathrm{HAp}$ content which did not clog the needle completely when extruded, indicating the removal of larger aggregates (Figure S3b). From the obtained data we concluded that the $0 \%$ and the $25 \%$ HAp content inks are suitable for printing with both the conical and straight $0.41 \mathrm{~mm}$ needles, whereas the $30 \% \mathrm{HAp}$ content ink would only be suitable to be printed with the conical $0.61 \mathrm{~mm}$ needle.

\subsection{Ink homogeneity}

To confirm a homogeneous distribution of HAp particles, we additionally performed TGA measurements to evaluate the solid particle content in different parts of the cartridge (Figure 6a). As shown in Figure 6b, TGA measurements revealed that, despite drying of the samples in a vacuum oven at $60^{\circ} \mathrm{C}$, there was still residual water (most probably bound) in the sample which evaporated in the temperature window between $50^{\circ} \mathrm{C}-200^{\circ} \mathrm{C}\left(2.5 \%\right.$ of the initial weight). Between $200^{\circ} \mathrm{C}-900^{\circ} \mathrm{C}$, a loss of $10.9-11.3 \%$ of the initial weight was observed, which is equivalent to the total dry polymer mass included in the ink. The residual $86.2-86.6 \%$ at $900^{\circ} \mathrm{C}$ represent the HAp particles. As evident from the TGA curves and from the insert, there was only a marginal difference between the sections within the printer cartridge in terms of the composition.
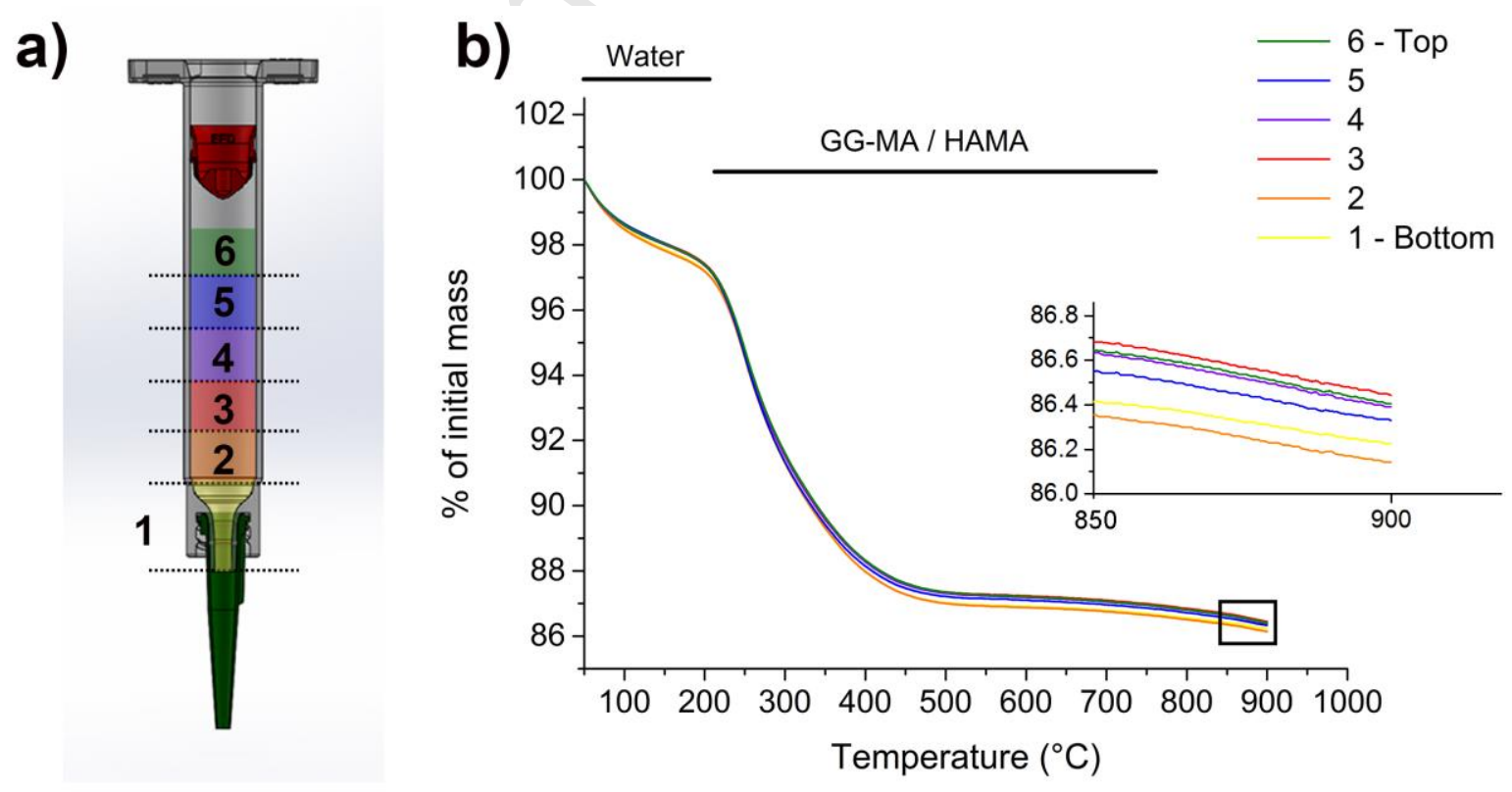
Figure 6: a) Schematic of the compartments within the printer cartridge which were evaluated with TGA. b) TGA measurement of different sections of a cartridge loaded with 30\% HAp content ink. The insert shows a magnified view of the weight differences between $850^{\circ} \mathrm{C}$ and $900^{\circ} \mathrm{C}$.

\subsection{Ink Printing}

To evaluate the influence of printing speed on the strand size, lines were printed from inks with different HAp content using a range of printing speeds. The ink with 30\% HAp content was printed using a $0.61 \mathrm{~mm}$ conical needle (Figure 7a) and the ink with 25\% HAp content was printed with a $0.61 \mathrm{~mm}$ conical (Figure $7 \mathrm{~b}$ ) and a $0.41 \mathrm{~mm}$ straight steel needle (Figure 7c) as these were found to be suitable for printing without any clogging. The lines were evaluated at three different locations and an average was taken from two lines. For the $30 \%$ HAp content ink, we found differences in the thickness of the lines when printed with ink originating from different compartments of the cartridge (Figure 6a). However, the effect was only present at lower printing speeds (800 mm/min, Figure 7a) and diminished at higher speeds. The effect was less pronounced for the $25 \%$ HAp ink (Figure $7 \mathrm{~b}$ ) when printed with a $0.61 \mathrm{~mm}$ conical needle. Differences were especially minimal at higher printing speeds (> $600 \mathrm{~mm} / \mathrm{min}$ ). 
a) $30 \% \mathrm{HAp}, 0.61 \mathrm{~mm}$ conical, $95 \mathrm{kPa}$

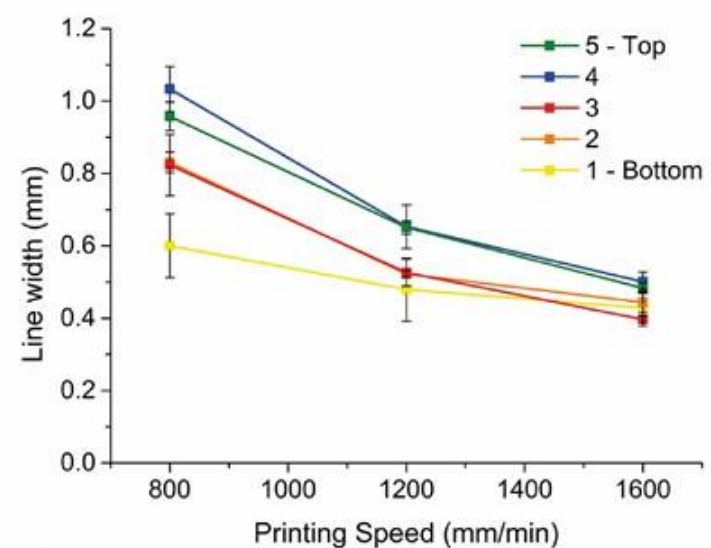

c)

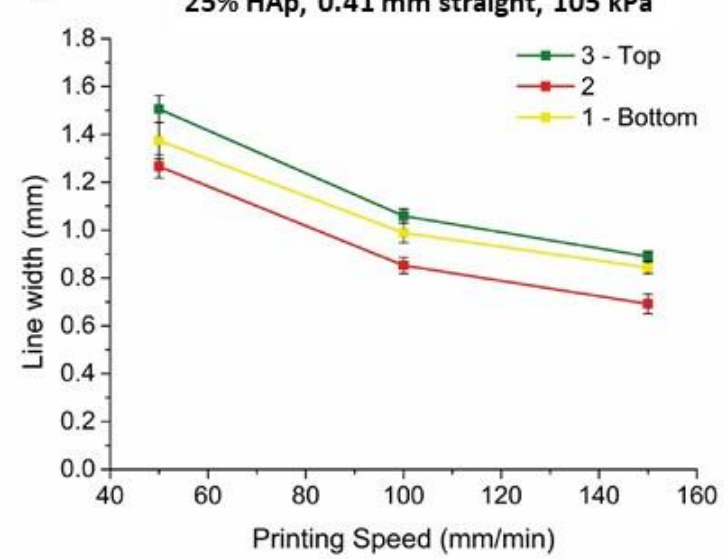

b)

$25 \%$ HAp, $0.61 \mathrm{~mm}$ conical, $55 \mathrm{kPa}$

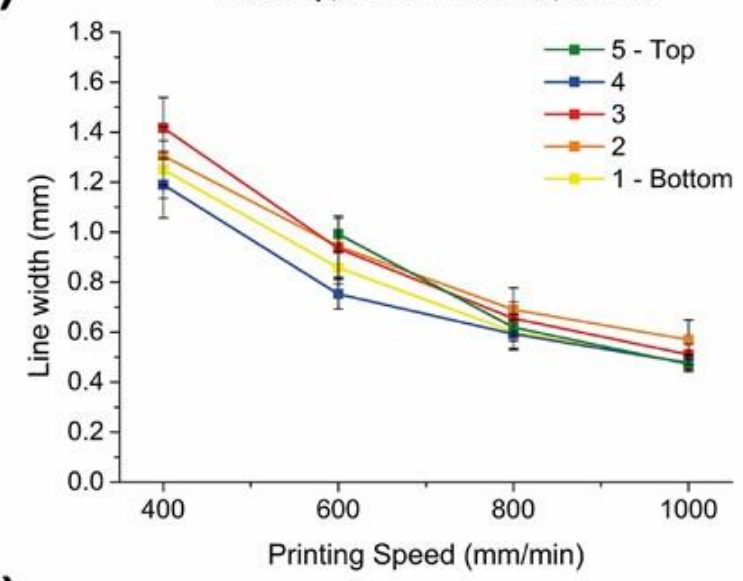

d)

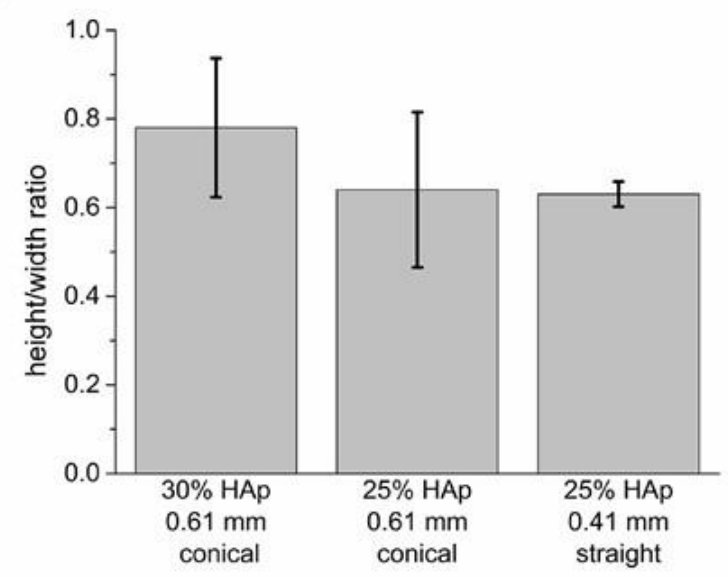

Figure 7: Line thickness at different printing speeds. a) 30\% HAp content ink, $0.61 \mathrm{~mm}$ conical needle, printed at $95 \mathrm{kPa}$ b) $25 \% \mathrm{HAp}$ content ink, $0.61 \mathrm{~mm}$ conical needle printed at $55 \mathrm{kPa}$ pressure and c) $25 \% \mathrm{HAp}$ content ink, $0.41 \mathrm{~mm}$ straight steel needle printed at $105 \mathrm{kPa}$ pressure. d) Width/height ratio average over the different printing speeds. The error bar indicates the variation of the width/height ratio between when using different printing speeds with the same needle.

When a $0.41 \mathrm{~mm}$ straight steel needle was used for the extrusion of the $25 \% \mathrm{HAp}$ ink, the printing pressure had to be increased to obtain a continuous flow of ink. The reason for this is the sharp transition from a large to a small diameter where the ink has to pass through. To minimize the effects of die swell (which increases with increasing pressure and flow rate of the ink), the lowest possible pressure for extrusion was chosen and printing was performed at low speeds (50-150 $\mathrm{mm} / \mathrm{min}$ ). There were still differences between the different parts of the cartridge (Figure 7c) but they did not follow a clear trend. Because the line thickness was less influenced by the ink's origin from within the cartridge, we chose to use the 25\% HAp ink for all future experiments involving printing. We also compared the ratio between the height and the width of the produced strands at different printing speeds (Figure 7d). The lower height/width ratio of the $25 \%$ HAp ink indicates that 
it continues to flow for a longer period of time following deposition, which means it recovers slower after shear, compared to the $30 \%$ HAp ink. When comparing the height/width ratio of the $25 \%$ HAp ink printed with either the $0.61 \mathrm{~mm}$ conical or the $0.41 \mathrm{~mm}$ straight steel needle, less variation was found between different printing speeds in the case of the $0.41 \mathrm{~mm}$ straight steel needle. Relaxation experiments on all the different inks revealed that with increasing HAp content, the stress within the materials (evident as normal force) is increased and that relaxation processes are slower (Figure S4). As the lines printed with the $0.41 \mathrm{~mm}$ straight steel needle were more homogeneous and less dependent on printing speed, we chose this setup for all future printing experiments to ensure consistent printing.

To assess the printability of the $25 \%$ HAp ink with the ideal needle geometry for this ink, cylinders with varying infill patterns were generated in Slic3r and postprocessed for the Biofactory in a custom written matlab postprocessor (Figure S5). Printed structures corresponded well with the STL design, having fully interconnected pores. Sharp corners however became more rounded caused by the speed at which the printer was moving.

To demonstrate the possibility of printing a bisphasic construct in a single process, grids of $10 \times 10$ mm were printed using the ink with 25\% HAp content and the ink with $0 \%$ HAp containing cells (Figure S6). The chosen interline spacing was $1.5 \mathrm{~mm}$ which resulted in pores with an average diameter of $794 \pm 65 \mu \mathrm{m}$, which is larger than the minimal pore size utilized for example in bone engineering [38]. For the bottom layer, 4 layers were printed up to a total height of $1.86 \mathrm{~mm}$ and horizontal pores were visible, which indicates that free standing strands could be produced with the $25 \%$ HAp content ink.

Because we used a 3D printing approach to create biphasic constructs, it was possible to print the cell containing layer directly on top of the bottom layer containing 25\% HAp. This circumvents the need to post-assemble the two layers in a separate step. The top layer consisting of the ink with $0 \%$ HAp content and containing the bovine chondrocytes nicely attached on top of the bottom layer after UV-crosslinking (Figure S6). The labelled bovine chondrocytes were visible by fluorescence microscopy and a clear interface between the two layers was observed (indicated by a dashed white line in Figure S6).

\subsection{Interlayer adhesion forces}

A push out test was performed to assess the bond strength of the interface between two printed ink layers containing $25 \% \mathrm{HAp}$ (Figure $8 \mathrm{a}$ ) as well as the bond strength between the inks containing $25 \%$ and $0 \%$ HAp (Figure $8 \mathrm{~b}$ ). For the push-out test, an outer ring of ink with $25 \%$ HAp content was 
printed and crosslinked for either 2, 4 or 8 minutes. The ring was then filled manually either with $25 \%$ HAp ink, $0 \%$ HAp ink, 1\% HAMA or 30\% Pluronic-dimethacrylate mixed with $5 \%$ chondroitin sulfate-methacrylate (PF-CSMA) followed by subsequent crosslinking via exposure to UV-light. The filling was then pushed out and the force required to break the interface was recorded. The pushout test revealed that there was no significant difference in the interfacial bond strength between outer ring and the filling if the outer ring was UV-crosslinked for 2 or $4 \min (27.5 \pm 5.3 \mathrm{kPa}$ and $24.7 \pm$ $2.9 \mathrm{kPa}$, respectively) before the filling was added and UV-crosslinked for another 2 minutes at the interface. After crosslinking the outer ring for 8 minutes, however, there was a significant drop (Oneway ANOVA, Bonferroni post-hoc, $p<0.05, n=4)$ in the interfacial bond strength $(18.5 \pm 3.0 \mathrm{kPa})$, indicating that the amount of available methacrylate groups on the surface of the outer ring was diminished. The amount is reduced to an extent where fewer methacrylate groups are available than could theoretically be crosslinked in the 2 minutes of UV-crosslinking at the interface.
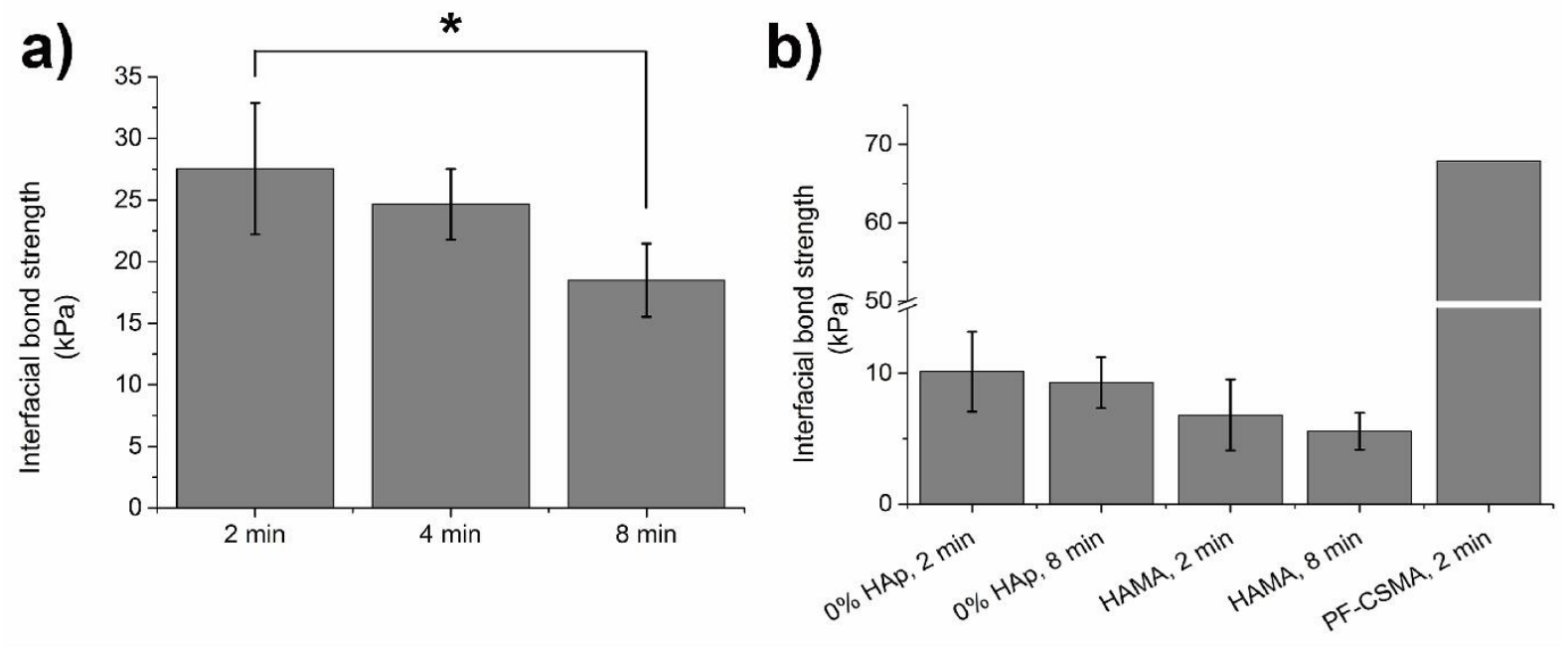

\section{c)}

d)
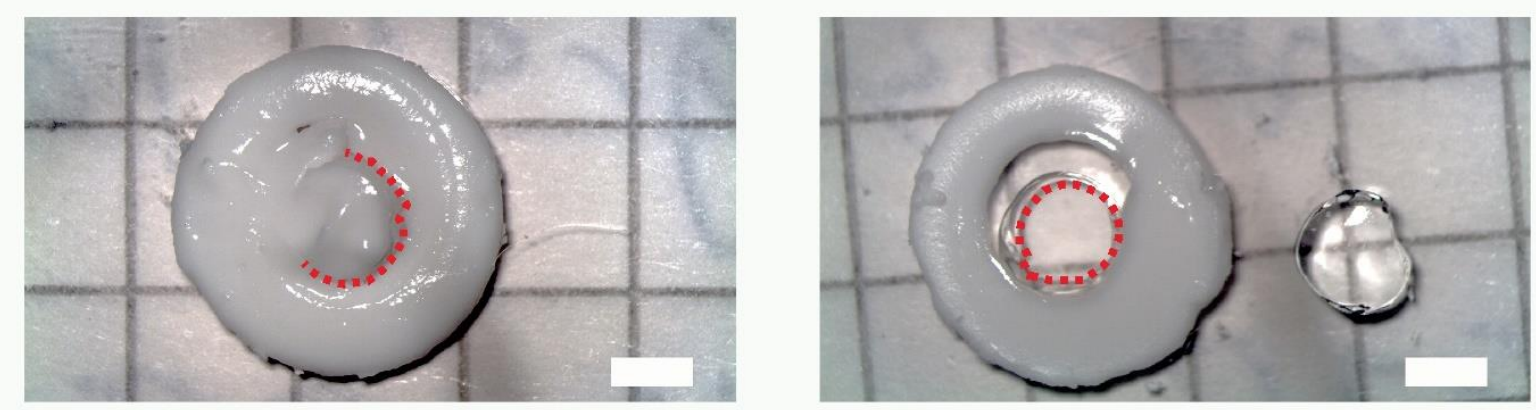

Figure 8: a) Interfacial bond strength of the interfaces between the outer ring and a filling of ink with $25 \% \mathrm{HAp}$. The outer ring was exposed to UV-light for 2,4 and $8 \mathrm{~min}$ before addition of the second layer. b) Interfacial bond strength between an outer ring of ink with 25\% HAp content and a filling of ink with $0 \% \mathrm{HAp}$ content, $1 \%$ HAMA and PF-CSMA. The outer ring was exposed to UV for either 2 or $8 \mathrm{~min}$. c) Ruptured interface between 
the outer ring and the filling of ink, whereas in d) the hydrogel representing the chondral layer (here HAMA) ruptured not at the interface but within the gel. Scale bar is $2 \mathrm{~mm}$.

It needs to be noted that, due to the fact that both the outer ring and the filling were made with $25 \%$ HAp ink, a clear distinction between rupture at the interface or within the filling was difficult to make (Figure 8c). However, the decrease in the interfacial bond strength, despite equal UV exposure times at the interface, suggests that the interface ruptured before the filling. For the interface between two different inks of a biphasic construct, we compared the interfacial bond strength between the $25 \%$ HAp ink and two different hydrogels that could potentially contain cells and serve as the top layer of a biphasic construct: $\%$ HAp content ink and HAMA ( $1 \% \mathrm{w} / \mathrm{v}$, Figure $8 \mathrm{~d}$ ). The interfacial bond strength for all interfaces was found to be lower than those for the interface between two 25\% HAp ink rings. However, no significant differences were found between 2 and 8 min UV-exposure to the outer ring (25\%HAp ink) (Figure $8 \mathrm{~b})$. This was the case for both the $0 \%$ HAp content ink (10.1 $\pm 3.1 \mathrm{kPa}$ and $9.3 \pm 2.0 \mathrm{kPa}$, respectively) as well as $1 \% \mathrm{HAMA}(6.8 \pm 2.7 \mathrm{kPa}$ and 5.6 $\pm 1.4 \mathrm{kPa}$ respectively). To test this hypothesis, we utilized a very stiff hydrogel, PF-CSMA, as the filling. We found that PF-CSMA did not break, but that instead the interface ruptured. The observed interfacial bond strength was $67.9 \mathrm{kPa}$, which is much higher than between two 25\% HAp ink layers. 


\subsection{Cell adhesion and viability}

When MSCs were placed on ink discs with different HAp contents, cell adhesion was observed in all samples but to different degrees with differences being observed between the inks containing $0 \%$ and the ones containing $25 \%$ and $30 \%$ HAp (Figure 9). On the 0\% HAp surfaces, MSCs grew in small isolated islands indicating a preference for cell-cell contact rather than cell-material interaction. The cells also did not display the spindle-like morphology characteristic for stromal cells. MSCs adhesion was higher on both $25 \%$ and $30 \%$ HAp containing ink compared to $0 \%$ HAp. Cells covered the entire substrate and were showing stromal cell morphology. This indicates that the addition of hydroxyapatite particles to the GG-MA/HAMA mixture improves the adhesion of stromal cells. The addition of HAp further seemed to increase the fluorescent background of the images which is why quantification of the surface coverage of MScs could not be reliably performed. Viability measurements using the MTS assay (Figure S7) as well as live-dead staining (Figure S8) revealed that the attached cells were viable as well, with the cells adherent on the 30\% HAp ink showing the highest viability after 3 days of incubation.

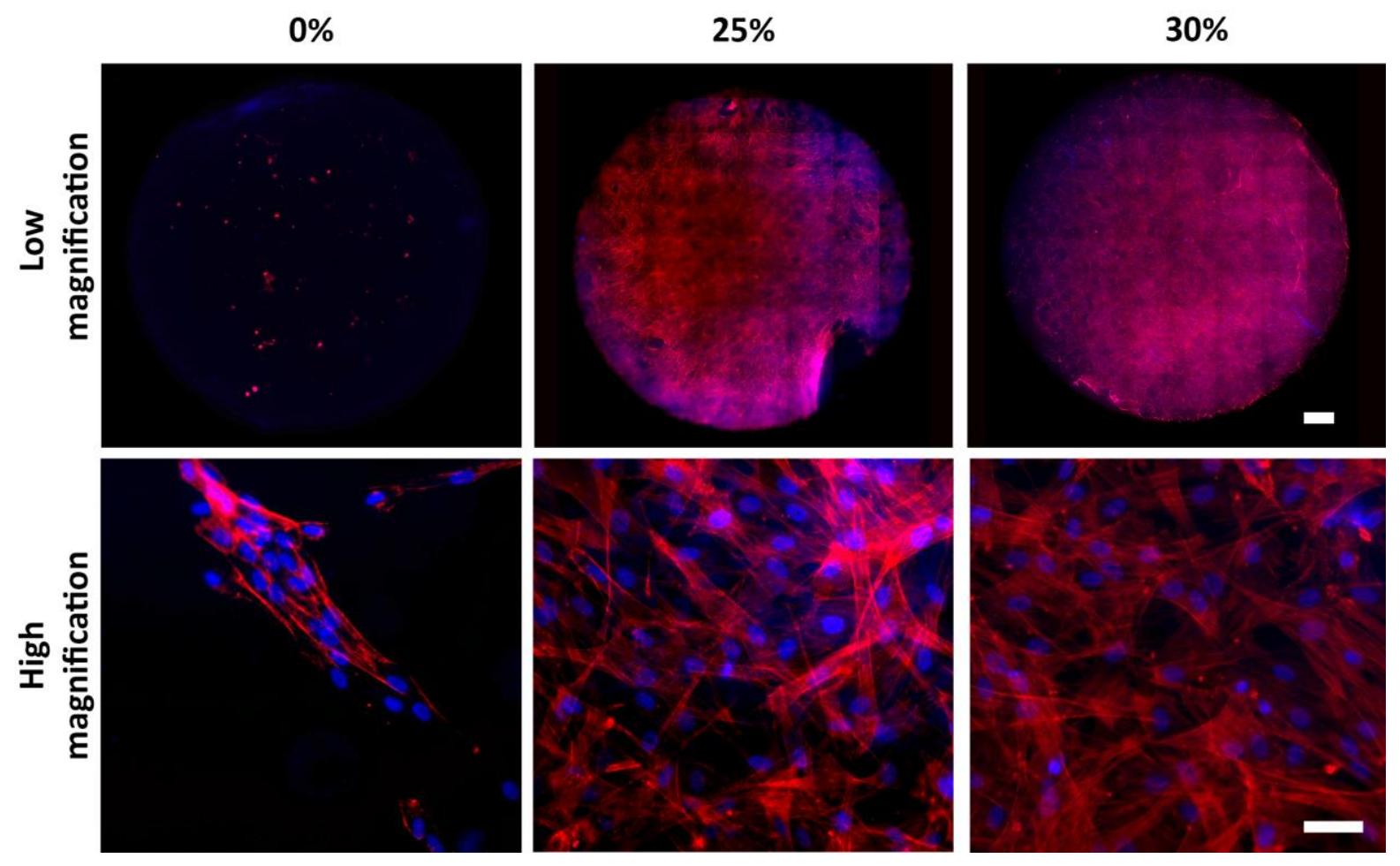

Figure 9: Adhesion of MSCs on $0 \%, 25 \%$ and 30\% HAp ink surfaces after 3 days in culture. Low magnification images are created from stitched $5 x$ magnification images and are showing a view of the entire surface. High magnification images show the spindle like morphology of the MSCs adhered to the surface. Scale bar is 500 $\mu \mathrm{m}$ for the low magnification and $50 \mu \mathrm{m}$ for the high magnification images. 


\subsection{Ink Surface}

To see if the addition of different amounts of HAp particles influences the surface topography, discs made from ink with either $0 \%, 25 \%$ or $30 \% \mathrm{HAp}$ content were created, exposed to $\mathrm{CaCl}_{2}$ overnight, placed in cell culture media for 7 days and analyzed with SEM (Figure S9). When no HAp was included in the ink $(0 \%)$, a number of smaller crystals were observed on the surface which could indicate the formation of hydroxyapatite [34]. Upon addition of HAp to the ink, these crystals were no longer present on the surface suggesting that the addition of HAp to the ink suppresses their formation. There was no obvious difference between the $25 \%$ and $30 \%$ HAp content ink surfaces as both displayed a rough topography with the HAp particles clearly visible.

\section{Discussion}

The production of large batch sizes of polymers for bioprinting applications using a robust synthesis method is a necessity to ensure consistent printing results. Here we presented the buffer mediated synthesis of GG-MA using the AMPD buffer. By avoiding manual control over the $\mathrm{pH}$, the risk for batch-to-batch variation was eliminated. Furthermore, higher degrees of substitution could be obtained compared to the conventional synthesis method when utilizing the same amount of GMA. By extending the duration of basic $\mathrm{pH}$ conditions in the reaction vessel, the transesterification of GMA with GG can continue and hence higher degrees of substitution can be obtained [25]. The obtained DS values were within a very narrow range of $1.72 \pm 0.20 \%$, which was also possible with batch sizes of $5 \mathrm{~g}$. Therefore, the buffer mediated synthesis offers a simple way to reproducibly create GG with different degrees of methacrylation (Figure 2a). We found, however, differences in the rheological properties of the inks corresponding to the different synthesis methods. We hypothesize that this is caused either by different distributions of the methacrylate groups along the GG-MA backbone or a preference of the carboxylic acid being modified rather than the primary alcohols, causing diminished double helix formation between chains [27, 39]. A lower amount of double-helices would result in fewer GG-MA aggregates [27] and, as a consequence, a lower, but still printable viscosity. Addition of AMPD to the GG polymer was not observed with NMR analysis and can therefore be excluded as a possible cause for the decrease in the viscosity. It needs to be 
evaluated if this $\mathrm{pH}$ controlled synthesis is also effective for other methacylation methods besides glycidyl methacrylate and if it also will influence rheological properties.

Freeze-drying is commonly applied after dialysis of derivatized polymers. While in the case of short (10-20 kDa) polymers, a powder is usually obtained after lyophilization, high molecular weight biopolymers produce sponge-like materials. These sponges have a very high volume to mass ratio and hamper dissolution with increasing concentration and make it difficult to blend with other polymers and/or hydroxyapatite particles. For storage purposes, it would be desirable to use cryomilling to create pre-mixed, dry powders that can be easily dissolved to create the final ink. However, while the low temperature during the cryomilling process aims to inhibit temperature mediated biopolymer chain scission, mechanical disruption of the chains still occurred as seen by SEC (Table S1), causing a drop in the viscosity of the ink (Figure 3) and lower final storage moduli upon UV-crosslinking (data not shown). Both the GG and GG-MA samples observed using SEC showed high Mw values in comparison to literature values [35], which we attributed to the fact that these measurements were performed at $35^{\circ} \mathrm{C}$, a temperature below the coil-coil formation temperature for gellan gum, leading to the formation of aggregates. Nevertheless, both the viscosities and the shear moduli of the UV-crosslinked inks produced with cryomilled polymers were still suitable for extrusion printing applications (Figure 4a and Figure 4b).

The inks showed shear thinning behaviour and appropriate stiffnesses after UV-crosslinking. It has been shown that Young's moduli in the range of $25-40 \mathrm{kPa}$ are suitable for the differentiation of stromal cells towards osteoblasts [40]. Under the assumption that the Poissons ratio is close to 0.5, the relation between the Young's modulus and the shear modulus is $E \approx 3 G$ which results in 151.8 $\mathrm{kPa}$ and $248.7 \mathrm{kPa}$ for the Young's moduli for the inks with $25 \%$ and $30 \%$ HAp content, respectively. It has been stated in the literature that the Young's modulus of mature osteoblastic collagenous bone environment is larger than $100 \mathrm{kPa}$ [40] and that stiffer substrates can cause upregulation of osteogenic genes [41]. Therefore, the moduli of the crosslinked inks would make them potentially 
suitable for the fabrication of a bone layer of a biphasic osteochondral graft where MSCs could adhere and differentiate into osteoblasts given that other requirements such as good cell adhesion and ideally osteogenic properties are fulfilled as well.

Figure $4 \mathrm{c}$ emphasizes the importance of standardized operating procedures for ink production as it shows that multiple high shear events (e.g. filling cartridges) can change the internal structure of the ink. Possible explanation for this behaviour would be the disruption of loose HAp aggregates, which are completely broken up after the second shear event, or potential alignment of non-spherical HAp particles or aggregates. This implicates that the processing history of the ink will influence its printing behaviour and care needs to be taken to ensure consistent printing results.

At any step during the production of inks for 3D printing applications, it is important to evaluate the inks for printability and homogeneity of the manufactured ink. Extrusion force measurement (Figure 5) in conjunction with thermogravimetric analysis (Figure 6) allowed us to check for local differences (aggregates) as well as global differences (HAp content throughout the cartridge) in the concentration of HAp particles in the ink. The constant force observed during the extrusion force measurements suggested that, apart from local heterogeneities in the form of aggregates, the HAp particles are distributed homogeneously throughout the cartridge. Hence, it can be concluded that mixing of the ink in the Thinky mixer setup was successful. From our experience we recommend the reader to always perform these two measurements during the development of novel particle containing inks, especially in cases where centrifugation is required to remove air bubbles introduced during cartridge loading, as this might cause concentrations of particles at the bottom of the cartridge. Aggregates were found in the $30 \%$ HAp ink which needles with a diameter $<0.61 \mathrm{~mm}$ were unsuitable for printing with this specific ink. Ultrasonication of the HAp particles before addition or the use of a 3-roll mill could help to break up larger aggregates and make a $30 \% \mathrm{HAp}$ loading suitable in the future. However, no difference was found in the HAp content throughout the printer cartridge (Figure 6). Despite this, we observed widening of the strands when later fractions of 
the cartridge were printed with the $30 \%$ HAp ink as seen in Figure 7. This would indicate inhomogeneity within the cartridge, which seemingly contradicts the findings from the extrusion force and TGA measurements. One key difference between the line assessment and the extrusion force measurement is that for the line printing, the ink is extruded out of the cartridge over the course of hours, whereas in the extrusion force measurements the entire cartridge was emptied within minutes. We therefore suspect a time-dependent change in the internal structure of the ink (relaxation) within the cartridge during the printing process, which would not change the composition but would alter the rheological behaviour of the ink. As this effect was not present for the $25 \%$ HAp ink, it indicates that the relaxation process in this composition is much faster than at $30 \%$ HAp content. leading to a lower height/width ratio or the printed lines. This was confirmed through relaxation experiments as shown in Figure S4. The faster relaxation process for the $25 \%$ HAp ink is further emphasized by the lower height/width ratio compared to the $30 \%$ HAp ink (Figure 7d) as the $25 \%$ HAp ink continues to flow for a longer period of time following deposition due to its already relaxed state.

Although large differences in line width for the 30\% HAp ink were present at low printing speeds, this was not the case at higher printing speeds. As the lines become thinner at higher speeds, less ink is deposited and as a consequence the observed relaxation (flow) of the material is less pronounced. When printing with a straight needle, the printing speed window for printing continuous strands was smaller, and therefore the speed had a smaller effect on the height/width ratio. It is often not obvious from the literature if our observations are a common phenomena or it is specific to our developed ink, but it seems to be highly recommendable to evaluate if the printing behaviour of an ink stays the same throughout a cartridge and with different needle geometries.

Due to more consistent printing throughout the cartridge, the $25 \%$ HAp ink composition was finally chosen for the printing of the biphasic constructs. Using our evaluation methods, we were also able to choose the right needle $(0.41 \mathrm{~mm}$, straight) for the printing of our ink. We were able to print 
biphasic constructs using the $25 \%$ HAp ink for the bottom layer and the $0 \%$ HAp ink for the top layer (Figure S6). These materials could potentially be used to create biphasic osteochondral constructs. Other polymers such as PCL or PLA that are used in fused deposition modelling (FDM) are also often employed in combination with $\mathrm{HA}[42]$ or other calcium phosphates such as $\beta-\operatorname{TCP}[43,44]$ to reach mechanical stiffness values closer to the human bone. However, apart from a few exceptions [45, 46] in many reports these FDM printed constructs do not allow the creation of a chemically crosslinked interface between the bone layer and a printable hydrogel containing cells and hydrogel biphasic constructs. Hence, hydrogel materials are often used for both phases of a biphasic construct [16]. The strength of the interface between layers was both dependent on the exposure time as well as the amount of available crosslinking sites. A shorter exposure time resulted in less covalent bonds between the outer ring and the filling, which lead to a weaker interface. On the other hand, in the case of PF-CSMA more covalent bonds could be established within 2 minutes due to the high amount of methacrylate groups providing for a stronger interface. Unfortunately, we were unable to measure the true interfacial bond strength between the $0 \% \mathrm{HAp}$ and the $25 \% \mathrm{HAp}$ ink as the ring made from $0 \%$ HAp ink ruptured before the interface. Nevertheless, we can state that the interfacial bond strength is stronger than $10.1 \pm 3.1 \mathrm{kPa}$ which is considerably stronger than, for example, the adhesion of fibrin glue to tissue [47-49]. The addition of HAp to the GG-MA/HAMA mixture improved the adhesion of MSCs to the ink and showed improvements with respect to viability when $30 \% \mathrm{HAp}$ was used (Figure S7). This was expected as GG itself does not possess any cell adhesion moieties and HAMA only possesses CD-44 adhesion sites. Similar effects have also been observed for other 3D printing materials such as PCL which makes the developed ink a promising candidate for further studies to investigate the influence of the HAp in the ink on the differentiation of adhered MSCs.

\section{Conclusion}

Reproducible large batch synthesis of GG-MA was successfully established and the influence of the synthesis and polymer processing on the rheological properties of an ink was evaluated. Through 
extrusion force measurements and TGA analysis it was possible to evaluate homogeneity of the ink which was important for the reproducible production of osteochondral constructs. These techniques should therefore be considered for standardizing experimental procedures for the quality control and assessment of inks for 3D printing applications.

\section{Declaration of Interest}

The authors report no conflicts of interest in this work.

\section{Acknowledgements}

This work was supported by ETH Research Grant ETH-23 14-1 and FIFA/F-MARC. We also thank Albrecht Berg for providing the photoinitiator LAP. We acknowledge support of ScopeM/Swiss Federal Institute of Technology ETHZ, specifically Stephan Handschin, for the help with the scanning electron microscopy. We further acknowledge Dr. Deborah Studer for the provided stromal cells utilized in this work. 
References

[1] L.G. Griffith, G. Naughton, Tissue engineering--current challenges and expanding opportunities, Science 295(5557) (2002) 1009-14.

[2] L. Guan, J.E. Davies, Preparation and characterization of a highly macroporous biodegradable composite tissue engineering scaffold, J Biomed Mater Res A 71(3) (2004) 480-7.

[3] D.J. Mooney, D.F. Baldwin, N.P. Suh, J.P. Vacanti, R. Langer, Novel approach to fabricate porous sponges of poly(D,L-lactic-co-glycolic acid) without the use of organic solvents, Biomaterials $17(14)$ (1996) 1417-22.

[4] H. Yoshimoto, Y.M. Shin, H. Terai, J.P. Vacanti, A biodegradable nanofiber scaffold by electrospinning and its potential for bone tissue engineering, Biomaterials 24(12) (2003) 2077-82.

[5] E. Sachlos, J.T. Czernuszka, Making tissue engineering scaffolds work. Review: the application of solid freeform fabrication technology to the production of tissue engineering scaffolds, Eur Cell Mater 5 (2003) 29-39; discussion 39-40.

[6] F.M. Klenke, Y. Liu, H. Yuan, E.B. Hunziker, K.A. Siebenrock, W. Hofstetter, Impact of pore size on the vascularization and osseointegration of ceramic bone substitutes in vivo, J Biomed Mater Res $A$ 85(3) (2008) 777-86.

[7] J. Groll, T. Boland, T. Blunk, J.A. Burdick, D.W. Cho, P.D. Dalton, B. Derby, G. Forgacs, Q. Li, V.A. Mironov, L. Moroni, M. Nakamura, W. Shu, S. Takeuchi, G. Vozzi, T.B. Woodfield, T. Xu, J.J. Yoo, J. Malda, Biofabrication: reappraising the definition of an evolving field, Biofabrication 8(1) (2016) 013001.

[8] N. Paxton, W. Smolan, T. Bock, F. Melchels, J. Groll, T. Jungst, Proposal to assess printability of bioinks for extrusion-based bioprinting and evaluation of rheological properties governing bioprintability, Biofabrication 9(4) (2017) 044107.

[9] K. Dubbin, A. Tabet, S.C. Heilshorn, Quantitative criteria to benchmark new and existing bio-inks for cell compatibility, Biofabrication 9(4) (2017) 044102.

[10] M. Kesti, C. Eberhardt, G. Pagliccia, D. Kenkel, D. Grande, A. Boss, M. Zenobi-Wong, Bioprinting Complex Cartilaginous Structures with Clinically Compliant Biomaterials, Advanced Functional Materials 25(48) (2015) 7406-7417.

[11] L.A. Solchaga, J.E. Dennis, V.M. Goldberg, A.I. Caplan, Hyaluronic acid-based polymers as cell carriers for tissue-engineered repair of bone and cartilage, J Orthop Res 17(2) (1999) 205-13.

[12] J. Kim, I.S. Kim, T.H. Cho, K.B. Lee, S.J. Hwang, G. Tae, I. Noh, S.H. Lee, Y. Park, K. Sun, Bone regeneration using hyaluronic acid-based hydrogel with bone morphogenic protein-2 and human mesenchymal stem cells, Biomaterials 28(10) (2007) 1830-7.

[13] J.R. Woodard, A.J. Hilldore, S.K. Lan, C.J. Park, A.W. Morgan, J.A. Eurell, S.G. Clark, M.B. Wheeler, R.D. Jamison, A.J. Wagoner Johnson, The mechanical properties and osteoconductivity of hydroxyapatite bone scaffolds with multi-scale porosity, Biomaterials 28(1) (2007) 45-54.

[14] S. Wust, M.E. Godla, R. Muller, S. Hofmann, Tunable hydrogel composite with two-step processing in combination with innovative hardware upgrade for cell-based three-dimensional bioprinting, Acta Biomater 10(2) (2014) 630-40.

[15] S.S. Kim, M. Sun Park, O. Jeon, C. Yong Choi, B.S. Kim, Poly(lactide-co-glycolide)/hydroxyapatite composite scaffolds for bone tissue engineering, Biomaterials 27(8) (2006) 1399-409.

[16] W. Schuurman, P.A. Levett, M.W. Pot, P.R. van Weeren, W.J. Dhert, D.W. Hutmacher, F.P. Melchels, T.J. Klein, J. Malda, Gelatin-methacrylamide hydrogels as potential biomaterials for fabrication of tissue-engineered cartilage constructs, Macromol Biosci 13(5) (2013) 551-61.

[17] S. Michna, W. Wu, J.A. Lewis, Concentrated hydroxyapatite inks for direct-write assembly of 3-D periodic scaffolds, Biomaterials 26(28) (2005) 5632-9.

[18] E. Hoch, T. Hirth, G.E.M. Tovar, K. Borchers, Chemical tailoring of gelatin to adjust its chemical and physical properties for functional bioprinting, Journal of Materials Chemistry B 1(41) (2013) 5675-5685. 
[19] B.J. Klotz, D. Gawlitta, A. Rosenberg, J. Malda, F.P.W. Melchels, Gelatin-Methacryloyl Hydrogels: Towards Biofabrication-Based Tissue Repair, Trends Biotechnol 34(5) (2016) 394-407.

[20] M.T. Poldervaart, B. Goversen, M. de Ruijter, A. Abbadessa, F.P.W. Melchels, F.C. Oner, W.J.A. Dhert, T. Vermonden, J. Alblas, 3D bioprinting of methacrylated hyaluronic acid (MeHA) hydrogel with intrinsic osteogenicity, PLoS One 12(6) (2017) e0177628.

[21] A.V. Reis, A.R. Fajardo, I.T.A. Schuquel, M.R. Guilherme, G.J. Vidotti, A.F. Rubira, E.C. Muniz, Reaction of Glycidyl Methacrylate at the Hydroxyl and Carboxylic Groups of Poly(vinyl alcohol) and Poly(acrylic acid): Is This Reaction Mechanism Still Unclear?, The Journal of Organic Chemistry 74(10) (2009) 3750-3757.

[22] J. Silva-Correia, V. Miranda-Goncalves, A.J. Salgado, N. Sousa, J.M. Oliveira, R.M. Reis, R.L. Reis, Angiogenic potential of gellan-gum-based hydrogels for application in nucleus pulposus regeneration: in vivo study, Tissue Eng Part A 18(11-12) (2012) 1203-12.

[23] J. Silva-Correia, J.M. Oliveira, S.G. Caridade, J.T. Oliveira, R.A. Sousa, J.F. Mano, R.L. Reis, Gellan gum-based hydrogels for intervertebral disc tissue-engineering applications, J Tissue Eng Regen Med 5(6) (2011) e97-107.

[24] J. Silva-Correia, B. Zavan, V. Vindigni, T.H. Silva, J.M. Oliveira, G. Abatangelo, R.L. Reis, Biocompatibility evaluation of ionic- and photo-crosslinked methacrylated gellan gum hydrogels: in vitro and in vivo study, Adv Healthc Mater 2(4) (2013) 568-75.

[25] Q. Li, D.-a. Wang, J.H. Elisseeff, Heterogeneous-Phase Reaction of Glycidyl Methacrylate and Chondroitin Sulfate: Mechanism of Ring-Opening-Transesterification Competition, Macromolecules 36(7) (2003) 2556-2562.

[26] L.F. Wang, S.S. Shen, S.C. Lu, Synthesis and characterization of chondroitin sulfate-methacrylate hydrogels, Carbohydrate Polymers 52(4) (2003) 389-396.

[27] D.F. Coutinho, S.V. Sant, H. Shin, J.T. Oliveira, M.E. Gomes, N.M. Neves, A. Khademhosseini, R.L. Reis, Modified Gellan Gum hydrogels with tunable physical and mechanical properties, Biomaterials 31(29) (2010) 7494-502.

[28] H. Shin, B.D. Olsen, A. Khademhosseini, The mechanical properties and cytotoxicity of cell-laden double-network hydrogels based on photocrosslinkable gelatin and gellan gum biomacromolecules, Biomaterials 33(11) (2012) 3143-52.

[29] H. Shin, B.D. Olsen, A. Khademhosseini, Gellan gum microgel-reinforced cell-laden gelatin hydrogels, J Mater Chem B 2(17) (2014) 2508-2516.

[30] M. Bohner, G. Baroud, Injectability of calcium phosphate pastes, Biomaterials 26(13) (2005) 1553-63.

[31] S. Wust, R. Muller, S. Hofmann, 3D Bioprinting of complex channels-Effects of material, orientation, geometry, and cell embedding, Journal of Biomedical Materials Research Part A 103(8) (2015) 2558-2570.

[32] T. Ahlfeld, G. Cidonio, D. Kilian, S. Duin, A.R. Akkineni, J.I. Dawson, S. Yang, A. Lode, R.O.C. Oreffo, M. Gelinsky, Development of a clay based bioink for 3D cell printing for skeletal application, Biofabrication 9(3) (2017).

[33] M. Kesti, M. Muller, J. Becher, M. Schnabelrauch, M. D'Este, D. Eglin, M. Zenobi-Wong, A versatile bioink for three-dimensional printing of cellular scaffolds based on thermally and phototriggered tandem gelation, Acta Biomater 11 (2015) 162-72.

[34] M. Kawashita, M. Nakao, M. Minoda, H.M. Kim, T. Beppu, T. Miyamoto, T. Kokubo, T. Nakamura, Apatite-forming ability of carboxyl group-containing polymer gels in a simulated body fluid, Biomaterials 24(14) (2003) 2477-84.

[35] H. Grasdalen, O. Smidsrød, Gelation of gellan gum, Carbohydrate Polymers 7(5) (1987) 371-393. [36] J. Mewis, N.J. Wagner, Colloidal suspension rheology, Cambridge University Press, Cambridge ; New York, 2012.

[37] T.G. Mezger, The rheology handbook: for users of rotational and oscillatory rheometers, Vincentz Network GmbH \& Co KG2006. 
[38] P. Duan, Z. Pan, L. Cao, Y. He, H. Wang, Z. Qu, J. Dong, J. Ding, The effects of pore size in bilayered poly(lactide-co-glycolide) scaffolds on restoring osteochondral defects in rabbits, J Biomed Mater Res A 102(1) (2014) 180-92.

[39] E. Miyoshi, T. Takaya, K. Nishinari, Rheological and thermal studies of gel-sol transition in gellan gum aqueous solutions, Carbohydrate Polymers 30(2) (1996) 109-119.

[40] A.J. Engler, S. Sen, H.L. Sweeney, D.E. Discher, Matrix elasticity directs stem cell lineage specification, Cell 126(4) (2006) 677-89.

[41] C.B. Khatiwala, P.D. Kim, S.R. Peyton, A.J. Putnam, ECM compliance regulates osteogenesis by influencing MAPK signaling downstream of RhoA and ROCK, J Bone Miner Res 24(5) (2009) 886-98.

[42] N. Xu, X.J. Ye, D.X. Wei, J. Zhong, Y.Y. Chen, G.H. Xu, D.N. He, 3D Artificial Bones for Bone Repair Prepared by Computed Tomography-Guided Fused Deposition Modeling for Bone Repair, Acs Appl Mater Inter 6(17) (2014) 14952-14963.

[43] J.L. Davila, M.S. Freitas, P.I. Neto, Z.C. Silveira, J.V.L. Silva, M.A. d'Avila, Fabrication of PCL/betaTCP scaffolds by 3D mini-screw extrusion printing, J Appl Polym Sci 133(15) (2016).

[44] J.C. Reichert, M.E. Wullschleger, A. Cipitria, J. Lienau, T.K. Cheng, M.A. Schutz, G.N. Duda, U. Noth, J. Eulert, D.W. Hutmacher, Custom-made composite scaffolds for segmental defect repair in long bones, Int Orthop 35(8) (2011) 1229-1236.

[45] K.W.M. Boere, J. Visser, H. Seyednejad, S. Rahimian, D. Gawlitta, M.J. van Steenbergen, W.J.A. Dhert, W.E. Hennink, T. Vermonden, J. Malda, Covalent attachment of a three-dimensionally printed thermoplast to a gelatin hydrogel for mechanically enhanced cartilage constructs, Acta Biomaterialia 10(6) (2014) 2602-2611.

[46] F. Chen, G. Hochleitner, T. Woodfield, J. Groll, P.D. Dalton, B.G. Amsden, Additive Manufacturing of a Photo-Cross-Linkable Polymer via Direct Melt Electrospinning Writing for Producing High Strength Structures, Biomacromolecules 17(1) (2016) 208-214.

[47] M.K. McDermott, T. Chen, C.M. Williams, K.M. Markley, G.F. Payne, Mechanical properties of biomimetic tissue adhesive based on the microbial transglutaminase-catalyzed crosslinking of gelatin, Biomacromolecules 5(4) (2004) 1270-9.

[48] M. Ishihara, K. Nakanishi, K. Ono, M. Sato, M. Kikuchi, Y. Saito, H. Yura, T. Matsui, H. Hattori, M. Uenoyama, A. Kurita, Photocrosslinkable chitosan as a dressing for wound occlusion and accelerator in healing process, Biomaterials 23(3) (2002) 833-840.

[49] Y. Nakayama, T. Matsuda, Photocurable surgical tissue adhesive glues composed of photoreactive gelatin and poly(ethylene glycol) diacrylate, J Biomed Mater Res 48(4) (1999) 511-21. 

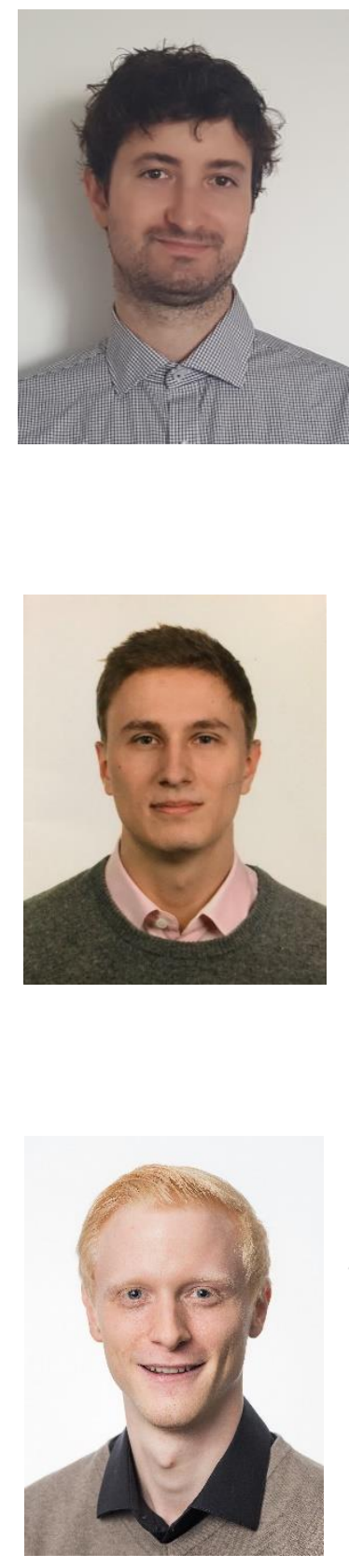

Marc Molnar has a Master of Science in Health Science and Technology from the ETH Zürich (Switzerland). In his Master thesis in the Tissue Engineering and Biofabrication Laboratory of Prof. Marcy Zenobi-Wong he focused on the development and characterization of a new bone ink for 3D bone printing. Currently he is a Scientific Expert at Geistlich Pharma AG (Wolhusen, Switzerland) and develops medical devices for regenerative medicine. He is an active member of the Swiss Society for Biomaterials and Regenerative Medicine (SSB+RM) Young Scientists and serves on the steering comittee for Health Science and Technology (HST) Alumni (ETHZ).

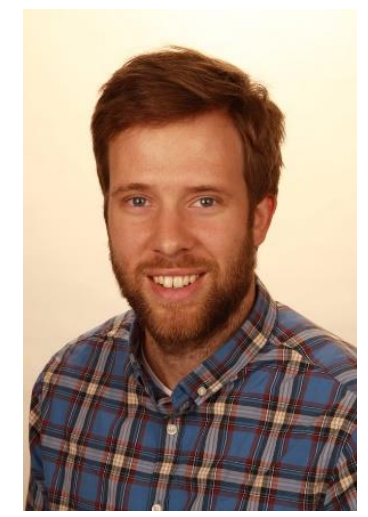

Sebastian Eggert has a Master of Science in Mechanical Engineering from the Technical University of Munich (TUM, Germany) and currently he is a 3rd year doctoral student at the Institute of Health and Biomedical Innovation (Queensland University of Technology, Australia). His research passion lies in interdisciplinary projects taking place at the borders where engineering and 
physics meet biology and medicine. Pursuing his passion for solving problems in medicine and improving biotechnology tools, Sebastian conducted research on cutting-edge technologies within the field of regenerative medicine, such as 3D printing of personalized implants, 3D bioprinting of tissue models, and organ-on-chip based instrumentation.
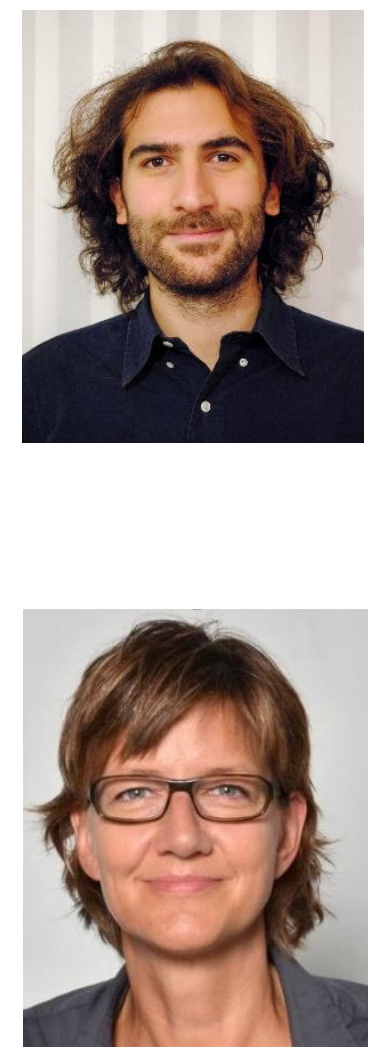

Marco R. Binelli obtained his Master's Degree in Materials Science from ETH Zurich (Switzerland), where he is also currently conducting his doctoral studies in the Complex Materials group.

His expertise and previous research experiences lie in bioprinting and soft materials fabrication; he is presently focused on soft bacteria-enabled materials and 3D printing technologies.

Katharina Maniura-Weber studied Chemistry at the Philipps-University in Marburg, Germany. She received her PhD at the University of Newcastle upon Tyne, UK, on research on cause and mechanisms of mitochondrial diseases employing in vitro cell models. Postdoctoral studies in Physiology departments of the Universities of Heidelberg and Cologne were on mitochondrial biogenesis and mitochondrial disease. At Empa she leads the Biointerface team, which works on biomaterials, surface modification, cell scaffold materials and the characterization of the biological response to materials. Since 2018 she is affiliated with ETH Zürich as Adjunct Professor in the Department of Health Sciences and Technology.

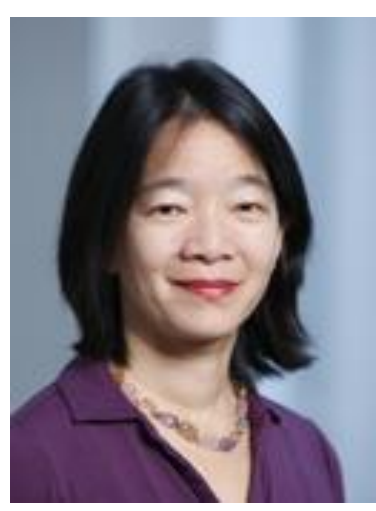

Dr. Marcy Zenobi-Wong is an Associate Professor of Tissue Engineering and Biofabrication at ETH Zürich, Switzerland. She obtained her PhD from Stanford University and did a post-doctoral fellowship at the University of Michigan. In 2012, she moved to the Department of Health Sciences \& Technology at ETH Zürich. Her research group focuses on the development of advanced biomaterials for tissue regeneration using various biofabrication technologies. She is currently President of the Swiss Society for Biomaterials and Regenerative Medicine and general secretary for the International Society of Biofabrication. She serves on the editorial board for Biofabrication and Advanced Healthcare Materials. 


\section{Declaration of interests}

$\bigotimes$ The authors declare that they have no known competing financial interests or personal relationships that could have appeared to influence the work reported in this paper.

$\square$ The authors declare the following financial interests/personal relationships which may be considered as potential competing interests: 